\title{
An Index of Plant Collectors in Brazilian Amazonia (")
}

Ghillean T. Prance (**)

\section{IN T ROD U C T ION}

During the course of my work as Curator of Amazonian Botany at the New York Botanical Garden, I frequently get requests for information about collectors in Brazilian Amazonia. This led me into the preparation of an index of collectors. This incex is not p esented as a comprehensive and complete work but rather as a compilation of all data available to me at the present time. Hence for some collectors great detail is given and fo: others only an outline simply depending upon the information which I was able to obtain. Additions and corrections will always be most welcome.

In cases where much of the information about a collector is already available in another easily accessible publication, I have merely drawn attention to this instead of repeating the same data.

Information has been gathered from a large number of sources. For collectors still living the degree of detail largely reflects how much information they were willing to communicate, and I am extremely grateful for the many replies to my letters asking for data.

\section{GEOGRAPHICAL RANGE OF INDEX}

For the present time I have restricted this index to collectors from Brazilian Amazonia only. That area is defined as the States of Amazonas, Acre, Pará, the western part of
Maranhão, the northern part of Mato Grosso and the Territories of Roraima, Amapá and Rondônia (see map) .

For the sake of uniformity I have used the modern names of cities which often appear on the labels of the older collectors under their former names. For example: Manaus (not Barra), Tefé (not Ega) etc.

A few collectors who have made large collections on the periphery of this area are mentioned, and it is hoped to treat the rest. of Amazonia at a later date.

\section{ACKNOWLEDGEMENTS}

I am extremely grateful to the numerous people that have provided data for this index. i should especially like to thank Dr. Paulo Cavalcante of the Museu Goeldi, Belém for furnishing much data about collectors from both herbaria in Belém, and Dr. William A. Rodrigues for data about collectors of the Instituto Nacional de Pesquisas da Amazônia. I am also grateful to Mr. Enrique Forero, and to my wife Anne E. Prance for much help in compiling this index, also to Mrs. Frances Maroncelli for typing all my drafts. This work is really the result of a New York Botanical Garden field program in conjunction with the Instituto Nacional de Pesquisas da Amazônia, Manaus, and I am grateful to the director and staff of the Instituto for much help, and to the National Science Foundation for grants GB 4641, GB-7356 and GB-18655 in support of the field program.

\footnotetext{
* - Produced in collaboration with the Instituto Nacional de Pesquisas da Amazônia Manaus, and the Museu Goeldi, Belém.

** - B.A. Krukoff Curator of Amazonian Botany. The New York Botanical Garden.
} 
THE COLLECTORS

OF BRAZILIAN AMAZONIA

ADAMS

AMAZONAS

$1940-1942$

Adams was manager of the Chicle Development Corporation in Manaus. He worked in collaboration with B.A. Krukoff and collected 79 numbers of latex bearing trees, mainly Sapotaceae. These collections are numbered as Adams $1-79$.

\section{ALBUQUERQUE, BYRON WILSON DE}

\section{AMAZONAS}

$1967-1969$

1967 Manaus $\mathrm{N}^{9}$ S $67 / 1-67 / 68,67 / 89-67 / 90$; 38 collections s.n.

1967 Mouth of Rio Branco 67/72-67/86

1968 Manaus 68/91-68/122 (plus 3 collections s.n.)

1969 Manaus 69/123 (plus 8 collections s.n.)

July 1969 Rio Solimões, Careiro Municipality, Lago de Janauacá 69/132-69/222.

Most collections have the collector's number which is proceded by the year of collection. Specimens deposited at INPA. Collections made by Albuquerque have been distributed under both of his forenames i.e. Byron \& Wilson, these may be detected by reference to the above list of collector's numbers.

\section{ALLEN, CAROLINE KATHRYN (1904 )}

Amazonas; Pará

1962,1966

\section{AMAZONAS}

Oct. 1966 Manaus \& vicinity, Manaus-Itacoatiara highway. Reserve Florestal Ducke (300-330).
PARÁ

Aug. 1962 - Belém \& vicinity (68-81)

NY; INPA (1966 collection only)

Allen collected exclusively Lauracea as part of monographic studies in that family.

ALUIZIO - Aluizio de Souza, J.

ALUIZIO DE SOUZA, JOÃO

Amazonas

1967 - Amazonas; Manaus, Reserva Fiorestal Ducke.

325 collections with collector's number, one without, deposited at INPA and duplicates distributed from INPA. Most labels have the collector's name as Aluizio.

ALVARENGA, Ten. M.

Terr. Roraima (Boa Vista); Pará (Cachimbo)

June, July 1965

Few collections only

Specimens at RB

A Lieutenant in the Brazilian airforce who made a few collections around some airports in Amazonia, the largest collection being the one in 1955 cited above.

ANDRADE, AYDIL GRAVE DE (1935 - ) ; see Emmerich, M.

ARAÚJO, VIVALDO CAMPBEL

Amazonas; PARÁ

$1964-1967$

Aug. 1967 - Amazonas; Itacoatiara (2 collections)

Oct. 1967 - Pará; Curuá-Una (21 co! lections)

Collections without collector's number but with INPA herbarium numbers; deposited at INPA, mostly distributed under the collector's name V. Campbell. 
ARCHER, WILLIAM ANDREW (1894-

PARÁ

$1942-1943$

July 1942 - March 1943 - Pará (mostly Belém and vicinity, about 100 numbers from Santarém and Rio Tapajós).

F,IAN,MO,NY,US

1000 numbers. Archer was assisted by Antonio da Silva.

ARENS, K.

AMAZONAS

1954 - 1955

Dec. 1954 - Amazonas; Manaus

Feb. 1955 - Amazonas; Manaus

3 collections without collector's number deposited (at INPA).

ARGENT, G.C.G.; See Royal Society

AUBRÉVILLE, A.

AMAZONAS; TER. RORAIMA

1958

AMAZONAS

Nov. 1958 - Manaus \& vicinity

TER, RORAIMA

Nov. 1958 - Boa Vista, Taiano, Foz Florestal

INPA,P

Aubréville's visit to Amazonia was primarily ecological with little collecting. Most of his collecting was with W. A. Rodrigues and under the latter collector's number series.

BACH, J.

PARÁ

$1903=$

1903 - Rio Xingú

MG
BAKER, CHARLES FULLER (1872 - 1927)

PARÁ

$1907-1908$

Dec. 1907 - July 1908 - Belém \& vicinity, especially grounds of Museu Goeldi

July 1908 - Rio Irituia

B,F,GH,IAC,K,L,MG,MO,NY,P,POM,U,US

Collection from Amazonia mainly of economic plants from vicinity of Belém. For biography see León, H. Elogio de Charles Fuller Baker. Acad. Cien. Med. Fis. \& Nat. Habana. 1928.

BAKSTA, P. CASIMIRO

AMAZONAS

1968 - Amazonas; Paricachoeira

6 collections with INPA herbarium numbers, deposited at INPA.

BALDWIN, JOHN THOMAS (1910 - )

Amazonas; Pará, Mato Grosso

Aug. 1942 - June 1944

\section{A,IAN,NY,SP,US}

Collections during much of his travels restricted to Hevea. The largest general collection was made from the Upper Rio Negro in 1944. The majority of the Hevea collection has noty et been distributed and is with Dr. Baldwin at Williamsburg, Virginia.

BARROSO, GRAZIELA, M.; see Royal Society

\section{BASTOS. ARTHUR MIRANDA}

PARÁ; TERr. Amapá

Oct. 1930 - Pará; Belém \& vicinity

Terr. Amapá; Rio Camaipi, Macapá etc. 1955 - 1956 - Terr. Amapá

Pará; Santarém \& vicinity

RB,IAN,NY,F

Bastos spent much time in Amapá in service of the Serviço Florestal and he was a forester rather than a botanist, but he macle over 500 collections in the course of his forestry work. 
B A T T EN-POOLE, Capt, ARTHUR HUGH (V.C.)

\section{AMAZONAS}

1891

Amazonas; Rio Ituxi, Rio Puciari, Upper Rio Purus.

Small collection at $\mathrm{K}$

\section{BAYERN, THERESE PRINZESSIN VON} $(1850-1925)$

AMAZONAS; PARÁ

Amazonas (Manaus, lower Rio Negro, lower Rio Solimões). Pará (Óbidos, Santarém, Belém).

July 1888

Part of the collection is at $\mathbf{M}$.

Few plants collected from Amazonia, but made more collections from southern Brazil.

\section{BLACK, GEORGE ALEXANDER (1910 - 1957)}

AMazonas; TERr. RORAIMA; PARÁ; TERr. AMAPÁ; MARANHÃo; TERR. RONDÔNIA

(1-52, 57-333 from Colombia with R. E Schultes; 694-784 from Peru \& Ecuador).

\section{Amazonas}

Tabatinga - 53-56

Manaus \& vicinity - 1150A; 1151A; 1161A; $1163 \mathrm{~A} ; 1174 \mathrm{~A} ; 1175 ; 1636-1640 ; 2856-$ $2860 ; 3034-3063$.

Manaus airport — 14076; 14088-14089; 14098 Manaus, Cachoeirinha - ? - 15353

Tefé, Rio Solimões — 1176-1635

Rio Negro; Camundé - 2396-2410

Rio Negro; Tapuruquara — 2411-2421; 28422854A; 2903-2910.

Rio Negro; Barcelos — 2422; 2444; 2889-2891

Rio Negro; Uaupés - 2445-2473; 2524-2541; 2545-2551; 2801-2805; 2825-2841; 2911-2913.

Rio Negro; São Felipe — 2542; 2729-2730; 2750-2753.

Rio Negro; "Morcego — 2543-2544; 2755-2797: 2882

Rio Negro; Parauari - 2552-2559
Rio Negro; Airão — 2893-2900

Rio Negro; Moura - 2901-2902

Rio Içana; Santarém - 2505-2523

Rio Içana; between mouth \& Tunui - 25602717 ; 2806-2824; 2855; 2864-2881; 28832888; 2892.

Bôca do Içana — 2718-2728; 2731-2749; 2754

Rio Içana; Camarão - 2798-2800

Rio Jamundá - 10801-10822

Cocodinho - 10823-10844

Aminarí, Assú — 10845-10873

TERR. RORAIMA

These collections were made between August 14 \& November 14, 1951.

Boa Vista - 12478-12627; 12650-12669; 13014 $13071 ; 13432 ; 13435-13440 ; 13725-13756$; $13759 ; 13789-13797 ; 13962-13981 ; 13984$. $13985 ; 13987-14023 ; 14026-14039 ; 14087$; 14090-14095.

Vicinity of Boa Vista - 12684-12731.

Road Boa Vista-Caracaraí - 13431; 13433. $13434 ; 13435-13462 ; 13463-13480 ; 13481$ 13513; 13558-13572.

Road Boa Vista to Colonia Fernando Costa - 13557 .

Mecajana - 12628-12649; 13072-13074; 13075 13160.

Igarapé Mecajana - 13982-13983; 13986; 14024-14025; 14096-14097.

Igarapé Carangá — 12670-12683.

Igarapé Caraná — 12732-12754; 12762-12795; 13757-13758; 13760-13776.

Rio Caomé - 12755-12761.

Rio Mucajaí; near crossing of Boa Vista-Caracaraí road; $12796-12802 ; 12820$; 12925; 12926-12979; 12803-12819.

Rio Mucajaí - 13334-13393; 13418-13430; 13514-13556; 13573-13724.

Road between Rio Mucajaí \& Caracaraí 13394-13417.

Rio Branco between Boa Vista \& Agua Boa - 12980-13013; 13161-13214.

Rio Branco; Fazendas Capela \& Bom Intento - 13215-13239; 13275-13333; 14040-14075.

Rio Branco - 13777-13788.

São Marcos - 13240-13274.

Serra do Cantá - 13798-13961. 
PARÁ

Belém \& vicinity — 692-693; 785-803; 813-849; 2350-2352; 2377; 2861-2863; 2914-2927; $3212-3216$; 3316-3319; 8036-8164; 81698171 ; 8546-8547; 8552; 8555-8569; 85718572; 8575-8576; 8621-8624; 8887-8891; $9368 ; 9740-9761 ; 10034-10035 ; \quad 10037$; 10039-10256.

Belém - Nov. Dec. 1950 - 10902-10926; ... $10936-10954 ; 10957-10960 ; 11702-11705$; 11752 ; 12023-12024; 12246-12247; 1244312459 ; $12465-12477$; 14078-14083; $14085-$ 14086 ; $14099-14172 ; 14238-14240 ; 14433-$ $14434 ; 15620$; $15645-15646$; $16102-16108$; 16112-16199; 16354-16357; 16359-16361; $16364-16370$; $16428-16429$; $16444 ; 16727-$ 16728 ; $16775-16781 ; 16817-16840 ; 16991$ 17001 ; 17003-17014; 17019-17020; 17022$17033 ; 18125-18139 ; 18474-18494 ; 18717$.

Oct. $\quad 1956-18825-18827$; 18835-18836

Nov. 1956 - 18891-18892

Nov. - Dec. 1956 - 18899-18941

Dec. 1956 - May 1957 - 18953-18960; 18966- 19417

June - July 1957 - 20011-20061

Santarém - 850-886; 964-966; 15621-15644; 18719-18744.

Oct. $\quad 1950-10257-10502$

July $1957-20062-20081$

Rio Utuquí - 887-930

Belterra, Rio Tapajós - 931-963; 967-979; $1151-1162 ; 1163-1174$

Rio Tapajós - 970-1150; 1641-1941; 2285-2349

Rio Pixuna - 1942-2005; 2032-2076

Rio Cuparí - 2077-2284

Rio Capim - 2353-2376; 2378-2386; 2394-2395

Rio Tinga - 2006-2031

Nengapí - 2387-2393

Vicinity of Antonio Lemos - 2928-3033

Rio Guamá - 3064-3211; 3320-3326; 3329$3362 ; 3363-3426$.

Dec. 1950 - 10927-10935; 10956

Nov. $1956-18837-18838 ; 18846-18851$

Vigia \& vicinity - 3217-3315; 8639-8835; 9763$9774 ; 14173-14174 ; 14216-14230 ; 14231$ 14234 ; $14235-14237$; 16729-16774; 16841-
$16989 ; 17002 ; 17015-17017 ; 18685$; 18795-18816.

Oct. - Nov. $1956-18818-18824$; 18828-18834

Dec. 1956 - 18942-18952; 18961-18965

Ilha do Marajó - 3427-3488; 3489-3639; 14241-14432

Rio Tocantins, Ipixuna, Jacundá, Tucuruí 7936-8035

Ananindeua - 8548-8551; 8838

Castanhal - 8584-8620

João Coêlho — 8625-8637; 16990-17018

Rio Paraca,uri - 8892-9367

Breves \& vicinity - 9775-9784; 9838-9849; 9851-9852; 10036

São Sebastião da Boa Vista - 9785-9789

Rio Mapuá — 9790-9837; 9850; 9853-9885; 10038

Cafezal - 10027-10033

Nov. 1950 - Morro de Óbidos - 10503-10523

Nov. 1950 - Rio Jamundá - 10524-10551; 10644-10689; 10690-10800.

Nov. 1950 - Faro, Lake Faro \& vicinity 10552-10631; 10632-10643; 10874

Nov. 1950 - Monte Alegre - 10875-10901

Dec. 1950 - Road João Coêlho to Vigia 14175-14215

Dec. 1950 - Cacaual Grande - 15354-15479; 15510-15557; 15574-15619

Dec. 1950 - Paraná do Tapará - 15480. 15509; 15558-15573

Dec. $\quad 1950$ - Rio Aurá - 16109-16111; 16371-16384

Dec. $\quad 1950$ - Rio Mojú - 16200-16353; 16362-16363

Dec. $\quad 1950$ - Maicurú - 18605-18620; 1862718633 ; 18659-18684; 18686-18716; 18718; 18746-18748; 18750-18778.

Aug. $\quad 1957-20082-20084 ;$ 20168-20169

Alenquer — 18621-18626; 18779-18794

Itaituba - 18749

Nov. 1956 - Highway BR114; near Santa Maria - 18839-18845

Nov. 1956 - Road to Irituia - 18852-18858

Nov. 1956 - São Miguel do Guamá - 18859; 18860-19890

May 1957 - Rio Cuminá, near Fortaleza - 19495 
May 1957 - Rio Jaramacurú — 19496-19508; 19509-19540

May 1957 - Rio Cuminá-Mirim 19541-19553

May - June 1957 - Region of Ariramba 19555-19905; 19906-19946

June 1957 - Oriximiná \& vicinity 19947-20010

August 1957 - Catera, Rio Maicurú - . . 20085-20167

TERr. Amapá

Oiapoque \& vicinity - 8172-8194; 8197-8409; $8440-8455$; $8457-8545 ; 8570$; $17602-$ 17623

Amapá - 9369-9632; 17680-18745; 18533-18535

Macapá — 9633-9732; 12248-12277; 1227812310 ; 12311-12343; 12363-12369; 14084; 18602-18604

Rio Araguari; Ferreira Gomes - 12344-12348; 18567-18571

Rio Araguari; Bom Retiro - 12349

Rio Araguari; 12370-12391; 12408-12442

Road Macapá-Clevelândia KM102 — 1235012355

Road Macapá-Clevelândia KM120 — 1235612362

Road Macapá-Clevelândia KM106 - 1239212407

Road Macapá-Clevelândia - 18495-18502; 18504-18508; 18519-18522; 18536$18556 ; 18572 ; 18574-18601$

Fortaleza de Macapá - 12460-12464

Tartarugal - 18503; 18523-18532

Porto Platon \& vicinity - 18509-18519; $18557-18566 ; 18573$

MARANHÃo

Imperatriz - 8165-8168

Carolina - 9733-9739; 9762

TERR. RONDÔNIA

Porto Velho \& vicinity - 14435-14696; 15348-15353

Guajará-Mirim \& vicinity — 14697-14728

Rio Guaporé from mouth to about Santa Cruz; Rio S. Miguel, also a few collections from Bolivian side of the Rio Guaporé - (Rio Iteñez in Bolivia) - 14729-15293.
Jaciparaná - 15347

First set at IAN, collections widely distributed from there, K, NY, US

Black's numbers are always preceded by the year of collections e.g. 51-12478.

The numbers missing from the series given here were mostly collected outside Brazil in North America.

Black was drowned while on a field trip in 1957.

\section{BOCKERMANN, WERNER}

PARÁ; ACRE; TERR, RONDÔNIA

1951 - 1956

PARÁ; Cachimbo (1955)

ACRE; Iquiri (1951), Tarauacá, Feijo (1956)

TERR. RONDônIA; Pôrto Velho (1954)

SP, some duplicates distributed to various herbaria .

He collected little material on the 1951, 1954,1956 , trips, but more in 1955. Material was collected without numbers and the numbers attributed to Bockermann were added by the São Paulo Herbarium. Bockermann is primarily a zoologist and much of his scrappy voucher material has been thrown away

BOYAN, RUFUS

\section{AMAZONAS}

1968

Amazonas; Guyana Frontier to Manaus. Nos. $172-300$.

Deposited at INPA and BRG

Also collected widely in Guyana.

BRAZÃO, UMBELINO; See Silva, N.T. da

BURCHELL, WILLIAM JOHN (1772 - 1863)

PARÁ; MARANhão

1828 - 1830

Dec. 1828 - Feb. 1830

PARÁ; Rio Araguaia, Rio Tocantins, Rio Mojú, Be'ém. Nos. 9092-10072

Maranhão; Carolina. Nos. 9026-9078

BR, GH, K, L, LE, OXF, P, US, W 
Collected much material from southern Brazil. For further details see Smith, L. B. \& R. C. Phytologia 14 (8) : 492-505. 1967.

BYRON - Albuquerque, B.W. de

CADMAN, G.

PARÁ

1919

$\mathrm{K}$

Small collection

CAMPBELL, V. - Araújo, V.C.

CAPUCHO, P.

PARÁ; lower Tapajós region at Fordlândia, Boa Vista.

1932 - 1933

F, IAN, U

About 300 numbers

Collected at the Ford rubber plant, ation from trees being felled during clearing operations for rubber tree planting. Capucho collected wood samples with many of his specimens. His collection was for B. E. Dahlgren of Chicago.

\section{CARDONA, FELIX}

Amazonas; Upper Rio Negro, Rio Padauiri 1943

NY, US, VEN

About 200 collections from Brazil. also collected widely in Venezuela.

\section{CASTELLANOS, A}

AMAZONAS

Jan. - Feb. 1963 - Manaus and vicinity, Ilha do Marapata, Manacapuru, Lower Rio Solimões, Beruri, Lower Rio Pu- rus, Rio Negro, Uaupés, Camanaus.

Without collectors numbers, INPA numbers 27509-27609.
CASTRO, R.A. de; See Royal Society

CAVALCANTE, PAULO BEZERRA

AMAZONAS; PARÁ

$1955-1970$

AMAZONAS

Jan. - Feb. 1959 - Rio Negro, Rio Uaupés, Taraquá

Jan. 1960 - Rio Negro, Ilha das Flores

March 1967 - Rio Negro, Rio Solimões, Manacapurú

June 1970 - Manaus (Nos. 2717-2724)

PARÁ

April - Sept. 1955 Belém \& vicinity

Sept. 1955 Vigia

March 1957 Marapanim, Marudá

May-June 1957 óbidos, Oriximiná, Rio Trombetas, Rio Cuminá, Rio Cuminámirim, Rio Jaramacarú \& Ariramba

June 1958 Vigia, Maracanã, Campo Martins, Pinheiro

July 1958 Marapanim, Marudá

June 1960 Óbidos, Rio Parú do Oeste

Nov. 1960 Belém

May 1965 Benevides

Feb. 1966 Benevides

Nov. - Dec. 1966 Santarém

Nov. 1967 Ilha do Mosqueiro

Feb. 1968 Rio Piriá \& Viseu

June 1968 Benevides

Sept. 1968 Marajó, Breves, Vila do Aramá

May 1969 Marabá, Serra dos Carajás

May-June 1969 Rio Acará \& Rio Mojú

June-Aug. 1969 Belém \& vicinity (Nos. 22742293)

Nov. 1969 Rio Jari, Monte Dourado (Nos. 2294-2316)

Nov. 1969 Belém (Nos. 2317-2320)

Feb. - March 1970 Parque Indígena do Tumucumaque, Alto Rio Parú do Oeste (Nos 2321-2609)

Apr. 1970 Marabá, Serra dos Carajás (Nos. 2610-2701)

May 1970 Belém (Nos. 2702-2708)

June 1970 Região do Salgado, São Caetano de Odivelas and Vigia (Nos. 2709 2716)

Collections at MG, INPA, 2321-2609 at NY 
CEZÁRIO, J .

AMAZONAS

1967

Amazonas; Manaus (3 collections)

Collections with INPA herbarium numbers and deposited at INPA.

CHAGAS, J.

AMAZONAS

1954 - 1959

1954 Amazonas; Manaus (332 collections)

1955 Amazonas; Manaus (693 collections)

1956 Amazonas; Manaus (400 collections)

1957 Amazonas; Manaus ( 94 collections)

1958 Amazonas; Manaus ( 87 collections)

1959 Amazonas; Manaus ( 36 collections)

Specimens deposited at INPA and duplicates widely distributed from there e.g. MCx. NY.

1954 - 1957 and part of 1958 - 1959 collections without collector's number but with INPA herbarium numbers. Part of 1958-1959 collections are Chagas Nos. $1-45$.

\section{COÊLHO, DIONISIO FERNANDEZ}

COÊLHO, LUIZ FERNANDEZ

\section{AMAZONAS}

1955 - $1959 ; 1966$ -

1955 Amazonas; Manaus (494 collections)

1956 Amazonas; Manaus (138 collections)

1957 Amazonas; Manaus (188 collections)

1958 Amazonas; Manaus ( 70 collections)

1959 Amazonas; Manaus ( 14 collections)

Apr. 1959 Amazonas; Rio Cueiras (18 collections)

Apr. 1959 Amazonas; lower Rio Negro, Igarapé Sumaúma (29 collections)

1966 Amazonas; Manaus-Itacoatiara road (4 collections)

1967 Amazonas; Manaus ( 4 collections)

1968 Amazonas; Manaus ( 92 collections)

1969 Amazonas; Manaus

Specimens in INPA \& duplicates widely distributed e.g. IAN, MG, NY.

Mostly collected without collector's numbers but with INPA herbarium numbers. 1958 collections included L. Coêlho Nos. 1 - 42 Many, of the earlier collections bore the collector's name Luiz with no reference to his surname.

\section{CORDEIRO, EDGAR}

TERR. RONDÔNIA

1952

TERr. RoNDÔNIA; Pôrto Velho \& vicinity IAN, NY

Agronomist \& Topographer who made about 400 botanical collections on his 1952 excursion to Rondônia with J. F. da Silva.

CORNER, EDRED JOHN HENRY (1906 -

AMAZONAS; PARÁ

1947 - 1948

AMAzonas; vicinity of Manaus

PARÁ; vicinity of Belém

IAN (vascular plants); R (fungi); CGE 
CORREA, WALTER MAURÍCIO

PARÁ

1955

1955 Pará; Santarém, Vila Curuaí

5 collections without collector's number, deposited at INPA.

COWAN, RICHARD SUMNER (1921 -

TERr. AMapÁ

1954

TERr. AMAPÁ

Nov. 1954 Serra do Navio, Pôrto Terezinha, Rio Amapari, Serra do Viado, Rio Araguari (Nos. 38051 - 38264).

Dec. 1954 Oiapoque airfield, Rio Oiapoque (Nos. 38625 - 38701).

F, GH, K, NY, US

The numbers are New York Botanical Garden series not the collectors. Also collected in Venezuela and the Guianas.

DAHLGREN, BROR ERIC (1877 - 1961)

AMazonas; Pará; Maranhão

1929,1938

Amazonas; Manaus

PARÁ; Rio Tapajós, Santarém, Boa Vista

MARANHÃo

\section{A, B, F, NY, POM, US}

In 1929, Dahlgren was accompanied by Emil Sella, a plant model maker and he collected mainly material for exhibition and economic plants. He travelled from Belém to Manaus, but most of his collecting was in the lower Rio Tapajós at the Ford rubber plantation in Boa Vista. He enlisted the help of P. Capucho \& R. C. Monteiro da Costa who collected herbarium specimens \& wood samples from trees being felled during clearing operations for rubber plantations. Capucho \& Monteiro da Costa sent material to Dâhlgren for about 3 years. This work from the rubber plantation by Dahlgren,
Capucho and Monteiro da Costa produced 1,503 herbarium specimens and 665 correlated wood samples.

Dahlgren álso made a short side trip to the state of Maranhão as well as collecting in northeastern Brazil and in many regions outside Amazonia.

DERLEY, O. A.

Pará; Santarém

1871

NY

Small collection originally deposited in Columbia College Herbarium and now at NY.

DIAS, J. S.

PARÁ

1943

IAN

Small collection.

DIONISIO - Coêlho, Dionisio F.

DREES, E. M.

AMazonas

1956

Aug. 1956 Ama.zonas; Manaus (1 collection without collector's number)

Oct. 1956 Amazonas; Benjamin Constant Nos. 1-48.

Specimens deposited at INPA.

DROUET, FRANCIS ELLIOT (1907 - )

PARÁ

1935

June - July 1935 Pará; Belém, Santa Izabel, Bahia de Marajó, Pôrto da Bazar, (Igarapé - Mirim), Carmo, Baião, Abaeté.

Several hundred collections of phanerogams, plus 60 algae.

Algae at $\mathrm{RB}, \mathrm{FH}, \mathrm{S}, \mathrm{L}$, and other herbaria. including the collector's personal herbarium.

Phanerogams G, F, US, NY and many others since plants were collected in sets of up to 30 duplicates. 


\section{DUARTE, APPARICIO PEREIRA}

AMAZONAS; TERR. RONDÔNIA

1962

Sept. 1962 Amazonas; Manaus, Barcelos

July. 1966 Amazonas; Manaus, Itacoatiara, Rio Preto

Sept. 1962 Terr. Rondônia; Pôrto Velho road to Cuiabá, Nova Vida.

RB

Duarte made only one short collecting trip to Amazonia in which he collected only a few plants in his own number series about Nos. $6880-7140$. He also collected extens. ively in eastern central and northeastern Brazil.

DUCKE, ADOLPHO (1876 - 1959)

amazonas; Terr. roraima, Pará, Terr. amapá, Maranhão, ACre, Terr, rondônia 1900 - 1958

Specimens deposited at: RB, MG, A, B, BM, EAC, F, G, GH, IAC, IAN, K, NY, P, PG, $\mathrm{R}, \mathrm{S}, \mathrm{U}, \mathrm{US}, \mathrm{W}, \mathrm{Y}$.

Most of Ducke's collections were made under the number series of the Institution for which he worked. Collections up to 1918 were collected under the Museu Goeldi (MG) numbers and collections from 1919 under the Jardim Botânico, Rio de Janeiro (RB). Care should be taken in citing Ducke numbers since many collections bear both MG and RB numbers. From 1937 he started his own number series for private distribution. This series is of about three thousand numbers.

The precise details of Ducke's excursions are not given here because they have already been published in great detail by Egler, Bol. Mus. Goeldi 18: 1 - 129. 1963; see also Archer, Taxon 11: $233-242.1962$.

\section{DUMONT, KENT PARSONS (1941 -- )}

AMAZONAE; TERR, RONDÔNIA 1968

AMAZONAS

July - 1968 - Manaus \& vicinity; Manaus-Itacoatiara highway
TERR. RONDÔNIA

June - July 1968 - Jaciparaná, Mutumparaná, Abunã (also in Bolivia, state of Pando, Bolivia Numbers 1-66).

INPA, NY, CUP

Collections of Fungi only, Dumont Brazilian numbers 1-263. Dumont collected while accompanying G.T. Prance's expeditions to the same area; he used a separate number series for the Brazilian and Bolivian collections.

\section{EGLER, WALTER ALBERTO}

Pará; Terr. amapá; Maranhão

1956 - 1961

PARÁ

1956 - Oct., Zona Bragantina \& Salgado

Nov.; Campos da Vigia

1957 - May 24 - June 8; Óbidos, Oriximiná, R. Trombetas, R. Cuminá, R. Cuminámirim, R. Jaramacurú, Campos de Ariramba

Sept. 29-30; Salinópolis

Oct. 24; Santa Izabel do Pará, Caraparú

1958 May 15; Campos da Vigia

1959 Jan. 18; Salinópolis July 12-20; Rio Cururú (Alto Tapajós)

1959 Aug. 26; Vigia, Campos do Caimbé \& Palha

Aug. 30; Marituba (Zona Bragantina), Granja Imperial

Sept. 1-4; Belém-Brasília Highway

1960 Jan. 28 - Feb. 17; Rio Cururú (Alto. Tapajós)

Mar. 8; Belém-Brasília Highway

Mar. 19-20; Castanhal, Curuçá \& Marapanim

May 23; Ananindeua

Sept. 3; Santarém

1961 Feb. 5-6; Zona Bragantina Mar. 10; Ilha do Marajó, Lago Ararí Apr. 6; Vigia

TERr. Amapá

1957 Oct. 28 - Nov. 5; Rio Araguarí. Rio Aporema, Serra do Navio

1960 Apr. 23-29; Oia.poque ,Clevelândia July - Oct; Rio Oiapoque, Clervelândia, 
Sto. Antonio — Nos. 47187-47199; 47224. 47239 ; 47635-47681; 47724-47741; 4776447772 (these numbers are in the New York Botanical Garden series).

1961 Mar. 31-Apr. 4; Macapá, Pôrto de Santana, Serra do Navio.

MARANHĀO

1958 Dec. 14 - 19

MG, NY

Egler lost his life on a field trip in a canoe accident in Terr. Amapá in 1961.

EITEN, GEORGE; See Royal Society

ELIAS - Elias de Paula, J.

ELTAS de PAULA, JOSÉ

AMAZONAS; PARÁ; TERr. AMAPÁ

1966 - 1967 - 1969

\section{AMAZONAS}

1966 - August - Amazonas; Pari Cachoeiras, Tio Tiquié $291-359$

1967 - Amazonas; Manaus Nos. 393.414; 419-432

1969 - Amazonas; Manaus, Manaus-Itacoatiara higway, Rio Negro, Baia Buiuçú 473-509.

PARÁ

1966 - March Belém \& vicinity Nos. 210-228 May - Região do Salgado (Curuçá, Marapanim \& Mosqueiro), Nos. 229-243 July - Zona Bragantina (Capitão Poço) Nos. 244-284

August - Castanhal \& S. Caetano de Odivelas Nos. 285-290

1969 - Vigia

TERr. AMAPÁ

1967 - Porto Platon Nos. 415-418

MG, INPA

The name of this collector varies on different labels, José Elias, J. E. de Paula, and most frequently just Elias. Numbers 210-359 have the first set at MG, 393-432 have the first set at INPA, and 473-509 have the first set at UB.

\section{EMMERICH, MARGARETE}

AMAZONAS; TERr. AMAPÁ, TERR. RONDÔNIA

$1961-1962$

AMAzonas

Nov. - Dec. 1962; Manaus to Taracuá

TERR. AMAPÁ

Jan. - Feb. 1961; Serra do Navio, Santana, Amapari

TERR. RONDÔNIA

1962 - Porto Velho (3 numbers only)

With Ancirade, Ardil G. de

About 500 numbers.

Deposited at $\mathrm{R}, \mathrm{HB}$

Emmerich collected with Andrade and all the collections bear two separate co!lector's numbers.

\section{EMYGDIO (DE MELLO FILHO), LUIZ}

Amazonas; PARÁ; ACre; TERr. RONdônIA; MATO GROSSO

Amazonas

1960 - Rio Branco to Bôca do Acre Manaus

PARÁ

1947 — Belém (with E.J.H. Corner \& Metraux)

1967 — Belém

ACRE

1960 - Rio Branco

TERR. RONDÔNIA

1961 - Cuiabá to Pôrto Velho

Mato GROSSO - 1961

200 numbers

Deposited at $\mathrm{R}$

Most collections bear the name Emygdio; but there are some labelled Mello Filho.

ERNANI - Ferreira, E.

\section{FARRISS}

PARÁ; Rio Maranhão, Rio Tocantins, Cametá Small collection in the mid 1800 s, exact date not known. $\mathrm{K}, \mathrm{M}$. 


\section{FERREIRA, ALEXANDRE RODRIGUES}

(1756 - 1815)

Amazonas; Terr. Roraima; Pará; Terr. RonDôNIA, MATO GROSSO

$1783-1792$

Oct. - Dec. 1783 - Pará; Belém, Ilha do Marajó

Jan. 1784 - Pará; Rio Tocantins, Cametá, Baião

1784 - Pará; Rio Amazonas

1784 - 1785 - Amazonas; Rio Amazonas; Rio Negro, Manaus, Barcelos, Uaupés, Rio Uaupés, São José de Mazabitenas, returned, via Rio Negro to Rio Brancc

Jan. - July 1786 - Terr. Roraima; Rio Branco, Rio Mereuini, Rio Catrimani, Rio Iniuiui, Rio Anaaaú, Caracarai, Rio Mucajai, Santa Bárbara, Fortaleza de S. Joaquim, mouth of Rio Tacutú, Rio Mahú, Rio Surumú, Rio Urari. coeira, Rio Pereré, Rio Parimé, Rio Marecé returned via Rio Branco.

Aug. 1786 - Mar. 1787 - Amazonas; Rio Negro, Barcelos, Rio Acará

Aug. 1787 - Aug. 1788 - Amazonas; Rio Solimões, Rio Negro, Manaus

Sept. - Dec. 1788 - Amazonas; Rio Madeira, Rio Aripuaná, Rio Mataurá, Rio Aianga-Tininga, Rio Manicoré, Rio Baeta, Humaitá to Rondônia.

Jan. - Sept. 1789 - Terr. Rondônia; Rio Madeira, Pôrto Velho, Rio Madeira via Abunã to mouth of Rio Beni, to Guajará-Mirim, to Rio Guaporé, Forte Príncipe de Beira, via Rio Guaporé to Mato Grosso.

Oct. 1789 - 1791 - Mato Grosso; Vela Bela, Cuiabá, Serra de S. Vicente, Xavier, Burutí, António Roiz, Santa Ana, Pilar, Santa Bárbara, Laurinhas, São Lourenço, Nova Coimbra, Rio Paraguai, Merim, Uberáva, Rio Aguapaí, Jaurú, Vila Bela .

Oct. 1791 - Jan. 1792 - Mato Grosso, to Belém via Rios Guaporé, Madeira, Amazonas.
Ferreira's expedition was without doubt one of the most interesting to explore Amaz nia since he was the first naturalist to collect plants in Brazilian Amazonia, yet he covered a greater itinerary than the majority of collectors cited here.

For further details about Ferreira see : França, C. : Doutor Alexandre Rodrigues Ferreria, História de uma missão científica ao Brasil no século XVIII. Bol. Soc. Brot. Ser. 2. 1 (1) : 65-123. 1922, where an identification ist of plants held at LISU is given.

Collections at LISU, $\mathrm{K}$ and $\mathrm{P}$. Many Ferreira collections were distributed without the collector's name many marked "ex Herb. Lusitanica, Pará". This is especially true of Paris collections of Ferreira. Paris has 1,114 sheets of Ferreira collections, and LISU has 1,213 .

In addition, most sheets are labelled Pará, but may have been collected throughout the region covered by his itinerary. This is the explanation for apparent geographical irregularities in his collections.

Some of the places cited in this itinerary did not exist as early as 1783-92 but names were added to make the itinerary more easy to follow.

Ferreira also made many zoological and anthropological collections

FERREIRA, ERNANI

AMAZONAS

$1955-1958$

Jan. 1955 - Amazonas; Rio Purus, Ipiranga, Vila Cruz, Tapuru, Chavina, Coá (44 collections)

1956 Amazonas; Manaus (4 collections)

1957 Amazonas; Manaus Nos. 57/1 - 57/178

Jan. - Feb. 1958 - Amazonas; Manaus Nos $58 / 179-58 / 186$

Apr. 1958 Amazonas; Codajás Nos. 58/187 $-58 / 286$

June 1958 Amazonas; Manaus Nos. 58/287 $-58 / 315$

July 1958 Amazonas; Travessia Manacapuru to Rio Negro Nos. $58 / 319-58 / 328$. 
1955-56 collections bear numbers of the INPA herbarium and 1957-58 collections the collector's number proceded by the year.

Specimens deposited at INPA.

FITTKAU, E. J.

PARÁ; MATO GROSSO.

$1962-1965$

Mar. - Apr. - 1962 - Pará; Rio Parú do Oeste, Tiriós Nos. 1-167

Sept. 1965 Mato Grosso; Parque Nacional do Xingú

1965 collections without collector's numbers but with INPA herbarium numbers; deposited at INPA. 1962 collections made with D. Coêlho.

\section{FONSÊCA, OLYMPIO DA}

\section{AMAZONAS}

Nov 1955 - Amazonas; Manaus

1 collection without number, deposited at INPA.

FORERO, ENRIQUE (1942 - )

Amazonas; Terr. Roraima; Acre; Terr. Ron. DÔNIA

$1967-1968$

1967 Amazonas

1967 Terr. Roraima

1968 Acre; Campinas

1968 Terr. Rondônia; Pôrto Velho to Jaru

INPA, NY, US, MG, K R, S, COL

About 300 numbers

Also collected with G.T. Prance in Amazonas (1967); Terr. Roraima (1967); Terr. Rondônia (1968).

FRANCISCO - Mello, Francisco

FREITAS, MARLENE

AMAZONAS

$1956-1968$

1956-1968 Amazonas; Manaus (10 collections)
Some collections with collector's numbers 1-6; others without collector's number but with INPA numbers.

Collections deposited at INPA.

FRÓES, RICARDO de LEMOS (1891 - 196 )

(Numbers 1-9889 were collected with or for B.A. Krukoff, and most of them bear the name Krukoff, since he was the principal collector. From 1942 onwards Fróes continued the number series for his own collectons, which are cited below).

Amazonas; ParÁ; Maranhão; Mato Grosso

Fróes Nos. 1-624 were collected for the Chicle Development Co., under the supervision of Krukoff and were from a large variety of regions and were confined to latex - bearing trees, mainly Sapotaceae.

AMAZONAS

March 1945 - Manaus \& immediate vicinity 20464-20548; 20550-20560; 2056220569; 20601-20605; 20607

March May 1945 - Rio Solimões, between Fonte Boa, São Paulo de Olivença \& Benjamin Constant 20549; 20570 20594

April 1945 - Fonte Boa \& vicinity 2060820664; 20666-20673; 20675 20682. Rio Solimões, São Paulo de Olivença $20683-20766$; $20787-20820 ; \ldots .$. 20846 ; 20850-20856; 20876; 20892 20914; 20923-20941; 20947-20949.

Apr.-May 1945 - São Antonio do Iça \& vicinity 20821, 20823-20830; 20832-20845; 20847-20849; 20857-20877; 20946

May 1945 - São Francisco do Iça 20831

Rio Solimões, Benjamin Constant 20878-20891; 20915-20921;

Rio Solimões, vicinity of São Paulo de Olivença 20904-20907;

Rio Solimões, Fonte Boa to Tonantins \& lower Rio Jutai 20950-20952; 20953; 20955-20958; 20959-20978; 20979-20999; 21000-21004; 21006; 21007-21010; 21048-21080. 
June 1945 - Rio Solimões; Fonte Boa to Tonantins \& lower Rio Jutai 2101121047.

Sept.-Oct. 1945 - Upper Rio Negro between Ilha Grande \& Cucuí, Rio Uaupés to Iauareté, Rio Aiary, Rio Içana 21081-21298; 21310-21313; 2131621320

Nov.-Dec. 1945 - Upper Rio Negro between Ilha Grande \& Cucuí, Rio Uaupés to Iauareté, Rio Aiary, Rio Içana 21311; 21314-21315; 21321-21389; 21394-21409; 21411-21489; 21515 21519; 21526-21536; 21533-21564

January 1946 - Manaus, Tarumã Falls 21565-21566

PARÁ

Sept. 1942 - Vicinity of Belém 20786

May 1943 - S. João do Araguaia 20272, 20275 July-Aug.-Oct. 1943 - Rio Tocantins mouth to São João de Araguaia 20268 20271; 20273-20274; 20277-20280; 20282; 20285-20287.

July-Sept. 1943 — Lower Rio Tapajós 20283; 20297-20298

August 1943 - Rio Araguaia 20276 Rio Itacayuna 20284

Oct.-Nov. 1943 - Lower Rio Y.ingú, Mata Vi toria, Altamira 20231; 20288-20298

Dec. 1944 - Vicinity of Belém 20561

Jan. 1945 - Rio Guamá 20265-20267; 20361 20451; 20595-20600

March 1945 - Santarém 20452-20462

Nov.-Dec. 1945 - Vicinity of Belém 20665; 20674-20767; 20784; 20785; 20822

\section{MARANHÃo}

1932 - 1933 - Rio Maracaçume 1700 - 2024

June 1944 - Rio Pindaré 20242-20264; 20302203z8; 20330-20360

São Luiz \& vicinity 20301

April 1946 - 21567-21595

MATO GROSSO

1944

Sept.-Oct. — Rio Tapajós 20299-20300

For his higher numbers I do not have as complete details and so it is presented differently. Nos. 22049-34939 were collected be tween April 1946 and June 1960.
April 1946 - June 1960

Amazonas 22039-22454

Amazonas; Rio Negro 22455-22910

Amazonas \& Terr. Roraima; Rio Branco \& Ric Negro 22911-23366

Pará; Rio Tocantins 23367-23611

Terr. Roraima \& Amazonas, Rio Juruá 21600 21682

Amazonas; Pará; Maranhão 23679-24078

Amazonas; Pará; 24322-26110

Amazonas; Manaus, Rio Negro, Rio Tarumă, Rio Urubú, Rio Tonantins, Alto Solimões \& Terr. Amapá; Região do Oiapoque 26111-26334

Amazonas; Pará; Terr. Amapá; Maranhão 26335-26858

Pará; Terr. Amapá; Amazonas 26900-27740

Pará; Belém I.A.N.; Maranhão; Amazonas 27741-28497

Maranhão; São Luiz; Pará; Amazonas 2849829553

Amazonas; Goiás 29554-30185

Pará: 30186-30622; 30962-31518; 31520-32789: $32800-32999 ; 33160-33210$

Maranhão \& Pará 30623-30805; 30806-30961

Pará; Santarém, Rio Curuá 33071; I-33093 XXIII

Amazonas 33000-33099, 33100-33159

Amazonas \& Minas Gerais $33280-34170$

Pará; Rio Gurupí, Rio Piriá, Viseu, Amazonas; Uaupés; Maranhão 34171-34934

Maranhão; Rio Maracaçume 34385-34931, $34934,34935,34938,34940,34941$. 34949

Pará; Belém 34932

Maranhão, São Luiz 34933

Pará; Belém-Brasília highway 34936, 34939 34941

Pará; Rio Tapajós 34937

First set of collections at IAN, duplicates widely distributed e.g. K,NY,SP,UC,US .

FROMM (TRINTA), ELZA

Amazonas; Manaus

Jan. 1963

190 collections

$\mathrm{R}, \mathrm{HB}, \mathrm{PEL}$

Fromm collected with E. Santos, J. C. 
Sacco, and L.A. Trinta. Each collector used his own number series, and therefore the plants bear 4 separate numbers. At the time of collecting her name was Fromm, but this has changed since she married one of the collectors and is now Fromm Trinta.

GIFFORD, D.R.; see Royal Society

GINZBERGER, AUGUST (1873 - 1940)

PARÁ

1927

Pará; Belém, Santarém

Primarily an ecologist but made herbarium collections on his expedition to Pará. Was accompained by $\mathrm{H}$. Zerny an entomologist.

Collections at W; some duplicates at F.

\section{GOELDI, ANDRÉ}

AMazonas; PARÁ

1902 - 1914

AMAZONAS

May 1903 - Manaus

June \& Sept. 1903 - Rio Purus

Feb. \& Mar. 1904 - Rio Purus

Jan. 1906 - Manaus

Feb. 1906 - Rio Purus

Pará

July - Nov. 1902 - Belém \& vicinity

Mar.-Apr./June-Sept. 1903 - Belém \& vicinity

Jan.-Feb. Apr. Dec. 1905 - Belém \& vicinity

Aug. 1906 - Belém \& vicinity

Aug. 1906 - Sto. Antonio do Prata

Oct. 1906 - Sta. Izabel S.M. do Guamá

Oct. 1907 — Belém \& vicinity

July-Sept. 1907 — Peixe-Boi

Sept. 1907 — Igarapé Açú

Sept. 1908 - Peixe-Boi

Feb. 1910 - Anajás

Oct.-Nov. 1913 — Rio Moju

Dec. 1914 - Belém \& vicinity

MG,RB

Care must be taken not to confuse André Goeidi with Emílio Goeldi former director of the Museu.
GOELDI, EMÍLIO

PARÁ

$1895-1907$

PARÁ

Oct.-Nov. 1895 - Cunany

August 1901 - Ilha do Mosqueiro

March 1907 - Peixe-Boi

MG,RB

Care must be taken not to confuse Emílio Goeldi collections with André Goeldi who collected much more extensively. E. Goelcii was primarily a zoologist who made a few botanical collections. He was also the directrr after which the Museu Goeldi is now named.

GOLDSMITH, F.B.; see Royal Society.

GOUVEA, J.

AMAZONAS

$1954-1956$

1954 Amazonas; Manaus ( 4 collections)

1955 Amazonas; Manaus (16 collections)

June 1955 Amazonas; Rio Preto, Bacabal (6 collections)

June 1955 Amazonas; Autaz-açu, Parana ds. Araça (3 collections)

1956 Amazonas; Manaus (1 collection).

Collections without collector's number but with numbers of INPA herbarium.

Collections deposited at INPA, duplicares distributed from there.

GUEDES, MANOEL

PARÁ; Terr. Amapá

$1896-1901$

PARÁ

June 1896 - Ilha das Onças

Feb-Aug. Sept. 1897 - Marajó

No of. collections

1897 - Belém \& vicinity

7

Sept. 1898 - Boa Vista 9

Sept.-Oct.-Dec. 1898 - Belém \& vicinity 27

Aug. 1899 - Castanhal 
Sept. Oct. 1899 - Marajó 38

June-July 1900 - Belém \& vicinity $\quad 7$

Nov.-Dec. 1900 - Belém \& vicinity 2

March 1901 - Utinga 13

Jan.-May \& Aug. 1901 - Belém \& vicinity 42

Aug.-Nov. 1901 - Breves \& Mexiana 291

Nov. \& Dec. 1901 - Belém east of Barra

Dec. \& Jan. 1901/02 - Marajó, Arary

Oct. 1901 - I .Mexiana

TERr. Amapá

Aug.-Sept. 1896 -

MG,RB, BM

M . Guedes was a gardener at the Museu Goeldi who also joined a few of the institution's collecting trips.

\section{GUEDES, TEMISTOCLES NEVES}

Amazonas; Pará

Amazonas; Manaus; Tarumã 20 - 36

Manaus; Estrada do Aleixo, Estrada do Paredão, 37 - 90

Porá-Mosqueiro, Prainha 1 - 19

Belém, IAN, Bosque Municipal 92 - 338

Belém, Mocambo 470 - 483

Municipality of Ananideua, Pireli 484-487

Near Igarapé Arapijó, Municipality of Breves $654-672$

IAN

Numbers $339-468 ; 490-653 ; 673-677$ were from the state of Ceará

HARLEY, R.M.; see Royal Society

HERINGER, EZECHIAS P.

TERR. RORAIMA

1965

Terr. Roraima; Tingui

$\mathrm{UB}, \mathrm{NY}$

Short excursion in April 1965. Also collected widely in Central Brazil.

HOEHNE, FREDERICO CARLOS (1882-1959)

Amazonas; Pará; Mato grosso

$1908-1914$.

1908 - Mato Grosso; Corumbá, São Luíz de Cáceres, Jacobina, Rio Jaurú.
1909 - Mato Grosso; Tapirapuã, Juruena, Serra dos Parecis, Utiarity, Rio Sacre, Rio Sepotuba, Tapirapuã, São Luíz de Cáceres.

1910 - 1912 - Mato Grosso; Corumbá, Rio Cuiabá, Serra de São Jerónimo, Rio Toquiari, Corumbá, São Luíz de Cáceres, Tapirapuã, Serra dos Parecis, Juruena, Serra do Norte, Rio Juruena: Amazonas; Rio Juruena : Pará; Rio Cururú, Rio Tocantins, Rio Amazonas.

1913 - 1914 - Mato Grosso to Tapirapuã

SP,R,A,B,BHMG,BM,BRSL,F,G,GH,IAC,K, LAS,M,MICH,MO,NY,POM,U,UC,US .

Hoehne was a member of the Rondon Commission expedition 1908 and the Telegraphic Lines Commission 1910-1914. For further details including a list of many of his numbers see Hoehne: Indice bibliográphico a numérico das plantas colhidas pela comissão Rondon, São Paulo, 1951. He collected 7594 numbers of his own on the commissions but only a small part of these were from Amazonia. He collected widely in the south of Brazil under the São Paulo (SP) herbarium number series.

HUBER, JACOB (JACQUES) (1867 - 1914)

Amazonas; Pará; ACre; Terr. Amapá $1895-1912$

PARÁ

Belém 1-97 (MG1-97); 107-242 (MG108-256); 488-551 (MG501-563) ; 552-561 (MG637646 ) ; 562-593 (MG 647-678); 1128-1157 (MG1259, 1262-1290)； 1474-1486 (MG 1639-1651); 1713 (MG2017); 1714-1715 (MG2022-2023); 1716 (MG2025); 17171739 (MG2027-2049); 1740 (MG2051); 1741-1749 (MG2053-2061); 1750-1753 (MG2063-2066) ; 1754-1756 (MG20682070); 1757-1759 (MG2072-2074); 17601762 (MG2076-2078); 1763-1770 (MG 2081-2088); 1771-1802 (MG2093-2124) 1803 (MG 2146); 1804 (MG2156); 1805 (MG2161); 1806 (MG2165); 1807-1808 (MG2167-2168); 1809 (MG2170); 1810 
(MG2173); 1811 (MG2175); 1812 (Mr; 2177); 1813-1814 (MG2599-2600); 1810 (MG 2605); 1816 (MG2609); 1817 (MG 2611); 1818 (MG2617); 1819-1820 (Mส 2621-2622); 1821 (MG2624); 1822 (MG 2631); 1994 (MG2988); 1995-1997 (MG 3005-3007); 1998-2000 (MG3014-3016); 2001 (MG3023); 2002-2003 (MG30263027); 2004 (MG3281) ; 2005-2019 (MG 3289-3303); 2020 (MG3309); 2021 (MG 3313) ; 2022-2023 (MG3324-3325); 20242025 (MG3397-3398); 2026-2034 (MG 3404-3412); 2035 (MG3414); 2036-2037 (MG3417-3418); 2038 (MG3660); 20412044 (MG3664-3667); 2045 (MG3676), 2046-2091 (MG3800-3845); 2092-2095 (MG3847-3850) ; 2096-2102 (MG38533859); 2103-2108 (MG4009-4014); 2109 2111 (MG4416-4418); 2112-2113 (MG 4167-4168); 2660-2663 (MG4843-4845); 2664-2677 (MG4967-4980); 2678-2680 (MG6876-6878); 2681-2684 (MG68886891) ; 2685-2692 (MG6893-6900) ; 26932695 (MG6963-6965); 2696-2701 (MG 6972-6977); 2705-2709 (MG6991-6995); 2710 (MG 6997); 2711-2727 (MG70067024); 2728-2744 (MG7029-7045); 27452746 (MG 7081-7082); 2747-2748 (MG 7235-7236); 2751 (MG7250); 2752-2757 MG7253-7258); 2758 (MG7260); 27592764 (MG7281-7286); 2765-2767 (MG 7302-7304); 2768-2771 (MG7317-7320); 2775 (MG7733); 2773-2786 (MG77417754) ; 2787-2788 (MG7774-7775); 27892790 (MG7779-7780)； 2829-2846 (MG 7847-7864); 2847 (MG8103); 2848 (MG 8167); 2849 (MG8171) ; 2850-5853 (MG 8173-8176) ; 2854-2860 (MG8223-8229); 2877-2908 (MG9321-9353); 2909-2910 (MG 9409-9410) ; 2911 (MG9827); 29122914 (MG10164-10166); 2940-2941 (MG 10861-10862); s. n. (MG11516-11522); s.n. (MG12179-12180); s.n. (MG12459).

PARÁ

Santa Izabel; 99-106 (MG99-107)

Marajó, Soure; 243-354 (MG257-370); 355-487 (MG371-500); 1823-1993 (MG2649-2819)
Rio Capim; 594-615 (MG679-700); 616-821 (MG701-904) ; 622-917 (MG905-1000)

Breves; 1444-1447 (MG 1609-1612); 1629-1672 (MG1823-1866); 1673-1712 (MG18671906) .

Monte Alegre; 1448-1471 (MG1613-1636)

Santarém; 1472-1473 (MG1637-1638); 29162939 (MG10435-10458)

Bragança railroad; 1487-1488 (MG1674-1675); 1489 (MG1676); 2915 (MG10344)

Bragança \& Ourém; 1490-1628 (MG1677-1818)

Peixe-Boi, Bragança railroad 2791-2828 (MG 7796-7833); 2861-2876 (MG8829-8844).

AMAZONAS

Amazonas 2114-2165 (MG4169-4220)

Sept. 1898 - Rio Solimões 1158-1161 (MG 1301-1304)

Apr. 1904 - Rio Purus 2280-2659 (MG43454729).

TERR, AMAPÁ.

Cunani 918-1070 (MG1001-1153); 10711091 (MG1160-1180); 1092-1127 (MG 1181-1220).

ACRE

Mar. 1904 - 2116-2279 (MG4236-4344)

Nos. 1162-1443 (MG1305-1585) were from

Peru. (Sept.-Dec. 1898)

MG,BM,US, etc.

HUNT, D.R.; see Royal Society

INPA (INSTITUTO NACIONAL DE PESQUISAS DA AMAZONIA)

Various earlier collections from Amazonas made by personnel from the Manaus Herbarium have no collectors name. Most of this material is from the vicinity of Manaus. Some sheets have as the locality B.A.M. This signifies Brazil, Amazonas, Manaus.

IRWIN, HOWARD SAMUEL JR. (1928 -)

ParÁ; Terr. Amapá

1960, 1961

PARÁ

July 1961 — Pará; Belém 45923-45925

Oct. 1961 - Pará; Belém 46710-46712

July 1961 — Pará; Arumanduba 45926-46039 
TERR. AMAPÁ

July-Oct. 1960 - Rio Oiapoque, Clevelândia, Santo Antonio etc. (Nos. 47133-47186; 47200-47223; 47240-47296; 47323-47371; $47374-47458 ; 47467-47532 ; 47539-47639$; $47682-47723 ; 47742-47763 ; 47773-48507$; 48581-48809; 48890-48892).

Some of these numbers were collected in French Guiana.

\section{PARÁ \& AMAPÁ}

July-Aug. 1961 - Rio Jarí (Nos. 46040-46709). NY,MG,F,GH,K,P,US,IAN

The numbers are the New York Botanical Garden Series. Irwin also collected widely in the Guianas and Central Brazil.

\section{JACCOUD, RENATO}

AMAZONAS; TERR. RORAIMA

1954

Aug.-Nov. 1954 - Amazonas; Manaus (4C collections)

Nov. 1954 - Amazonas; Ilha do Catalhão (5) collections)

Oct.-Nov. 1954 - Terr. Roraima; Normandia \& Socó (32 collections)

Collections are without collector's number, but bear number of the INPA herbarium Manaus. Collections deposited at INPA.

JOBERT, CLEMENS

A.MAZONAS; PARÁ; Maranhão

$1877-1878$

$\mathrm{P}, \mathrm{R}$

Accompanied C.A.W. Schwacke on his 1877-78 expedition from Maranhão through Pará and Amazonas to Peru. A few collections of Jobert's are separate from those of Schwacke.

JUNK, W.

AMAZONAS; PARÁ

$1967-1968^{\circ}$

Oct. 1967 -Amazonas; Careiro (6 collec tions)
Jan. 1968 - Amazonas; Manacapuru (5 collections)

March 1968 - Amazonas; Costa Baixa, Ric Solimões (4 collections)

May 1968 - Amazonas; Lago Xiborena (? collections)

July 1968 - Amazonas ;Lago Paru, Rio Solimões ( 6 collections)

June 1968 - Pará; Bôca do Rio Tapajós (2 collections) .

All collections without collector's number but with number of INPA herbarium, deposited at INPA.

KILLIP, ELLSWORTH PAINE (1890 - 1968)

Amazonas; Pará

Oct.-Nov. 1929

Amazonas; Manaus 30001-30205

Amazonas \& Pará; journey Manaus to Belém 30206-30231

Pará; Belém, Ilha Mosqueiro. Gurupá 3023230666 .

US,NY

Killip was accompanied by A.C. Smith . The Killip \& Smith work in Brazil was mainly in search of sources of the fish poison 'Timbo'. See : Killip, E.P. \& Smith, A.C. Botanical trip to eastern Peru and Amazonian Brazil. Bull. Pan. Am. Union, Washington 64: 997-1009. 1930.

KOCH, CHRISTIAN THEODOR (b. 1872)

AMAZonas; MATO GRosso

1899 - 1903

1903 Amazonas; Rio Negro to São Gabriel, São Felippe, Rio Içana, Rio Aiarí, Rio Caiari.

1904 Amazonas; Serra de Curicuriarí, Uanpés, Rio Tiquié, San Marcellino, Rio Caiari, Uaupés, Rio Cudiarí.

1905 Amazonas; Rio Cuiarí, Uaupés, Rio Tiquié, Piraparaná, Rio Solimões.

1899 Mato Grosso

$\mathrm{B}$, most of his plants were lost when the Berlin herbarium was destroyed in World War II. See also Urban (1906) . 
KRUKOFF, BORIS ALEXANDER (1898-

Amazonas; Pará; Maranhão; ACre; Território DE RONDÔNIA; MATO GROSSO

1931 - 1932 Amazonas; Rio Solimões 1271-1284

Amazonas; Rio Juruá 4525-5209

Amazonas; Acre; basins of Rio Purús,

Rio Ituxí \& Rio Marmelos 5812-5862

Pará; Rio Tapajós, Rio Cupari, plateau between Rios Xingú \& Tapajós 1014-1268.

Terr. Rondônia; Calama, Rio Machadc, Igarapé Jatuarana 1286-1693

1933 Maranhão \& Goiás; mostly Rio G:ajahú \& Tocantins 2028-2081.

Acre; Upper Jurupary (trib. of Rio Embira); Basin of Rio Purus, Rio Macauhan, Rio Iaco 5210-5811.

1934-1935 Amazonas; Rio Madeira, Manicore, Três Casas, Humaitá, Livramento, Rio Ipixuna 6034-7290.

1935 - 1936 Amazonas \& Pará; various localities 7501-7910.

1936-1937 Amazonas; Manaus,. lower Ris Negro, Rio Solimões, São Paulo de Olivença 7911-9124.

NY,US,A,BM,F,IAN,K,LE,MICH,MO,P,S, RB

For further details see Tropical Woods 65: 31 - 35. 1941.

Note Nos. 1314-1693 are labelled as from the state of Mato Grosso and 1286-1313 are labelled Amazonas. These were in fact collected in Territorio de Rondônia.

\section{KUHLMANN, JOÃO GERALDO}

AMAZONAS; TERR. RORAIMA; PARÁ; MATO Grosso; TERR. RONDÔNIA

Oct. 1914 - Feb. 1915

Mato Grosso; Cuiabá, Paratinga, Rio Arinos, Rio Juruena, Pará; Rio Tapajós, Santarém.

Mar. - Aug. 1918

Mato Grosso; Diamantino, Parecis, Três Jacús, Ponte de Pedra, Salto Belo, Salto Utia-

- rití, Juruena, Nambiquares, Campos Novos da Serra do Norte. TERR. RONDôNIA; José Bonifácio, Ariquemes, Rio
Jamarí. Amazonas; Rio Madeira, Pasto Grande to Manaus.

Nov. 1918-Feb. 1919

Amazonas; Manaus, Rio Madeira, Pasto Grande. Terr. RondônIA; Foz do Jamarí, Ariquemes, Cachoeira de St. Cruz, Urupá, Cataqui-Iamain, Rio Cantário, return by same route in reverse.

Kuhlmann collected 2507 numbers (Nos. 1-2507) during the three expeditions listec? above which were all part of the Commission of Telegraphic Lines. In addition to the Telegraphic expeditions Kuhlmann made other trips to Amazonia where he collected in a different number series also starting at 1 . Many of his plants from these trips were collected under the Rio de Janeiro Botanical Garden (RB) number series, some duplicatcs of Kuhlmann's own numbered collections were distributed with just the RB number. For this reason it is always better to cite the $R$ ? number with Kuhlmann's collections. His other expeditions to Amazonia were as follows. Dec. 1912 - Nov. 1913

Terr. Roramma; Boa Vista, Bomquerer. Vista Alegre, Rio Branco.

1923 - 1924

TERR. RONDÔNIA - Ouro Preto, Rio Pacaás Novas. Amazonas; Rio Madeira to Manaus. PARÁ; Rio Tapajós, Santarém.

$\mathrm{RB}, \mathrm{SP}, \mathrm{K}, \mathrm{US}$

Kuhlmann also collected extensively in southern Brazil.

\section{KUHLMANN, MOISÉS}

PARÁ

1959

PARÁ; Belém \& vicinity, Belém-Brasília higway.

with S. Jimbo

352 numbers, numbered 1-352. Kuhlmann started a new number series for his collection with Jimbo. His other extensive number series is all from southern Brazil.

SP,IAN (much material still undistributed) 
LABROY, O.

AMAZONAS; PARÁ

1906

$P$

Travelled from Belém to Manaus and a few other localities such as the lower Rio Xingú, on a study of rubber (Hevea brasi. liensis) and other latex plants. He made a few general collections in the course of this study.

LANGENHEIM, J. H.

AMAZONAS, Pará

Oct. 1969

Amazonas; Manaus and vicinity

PARÁ. Santarém

All collections of Hymenaea collected with W.A .Rodrigues, Nos. 5611 - 5629.

\section{LEDOUX, PAUL VINCENT DÉSIRÉ (1898 - )}

PARÁ; TERr. Amapá

IAN, MG, NY

Ledoux has collected widely in Pará \& Terr. Amapá over the past 20 years. At the time of this writing I have been unable to obtain his itinerary.

LIMA, DARDANO DE ANDRADE (1919 - )

Amazonas; Pará; Mato grosso

May-July 1966 Amazonas; Humaitá (150 numbers IPA)

Aug.-Nov. 1948 Pará; Belém \& vicinity (29 numbers, IAN, IPA)

Apr.-May 1953 Pará; Serra Itauajari, Monte Alegre (240 numbers IPA, IAN)

1958 Mato Grosso; Aragarças, Xavantina, Rio Mortes; Capitão Vasconcelos, Xingú National Park (300 numbers IPA, $\mathrm{K}, \mathrm{RB}$ ) .

Also collected with G.A. Black \& with J.M. Pires in their number series.

Most collections have the collector's name cited as A. Lima, The numbers are preceded by the year of collection.
LIMA, F. Q.

Pará; Maranhão

1908 - 1918

PARÁ

Sept. \& Dec. 1908 - Zona Bragantina

Jan. \& Feb. 1909 - Zona Bragantina

May 1909 - Zona Bragantina

Jan. 1910 - Viseu

Mar. \& June 1910 - Zona Bragantina

Aug. 1910 - Acará

Sept. 1910 - Zona Bragantina

July 1911 - Zona Bragantina

Oct. 1912 - Rio Tocantins

Nov. 1914 - Zona Bragantina

Feb. 1916 - Cametá

July 1917 - Rio Tapajós

Mar. 1918 - Santa Izabel

MARANHÃo

Apr. \& May 1909

$\mathrm{MG}, \mathrm{RB}$,

Collector of the Museu Goeldi whose plants were numbered in the Museum series.

LIMA, J. AMÉRICO

TERR, RORAIMA

1962

Feb.-Mar. 1962 Terr. Roraima; Boa Vista (13 collections)

Specimens without collector's number but with INPA herbarium numbers. Collection made with F. Mello \& deposited at INPA.

LISBOAA, ARROJADO

Maranhão

Aug. - Sept. 1909

MG,RB

Geologist who also collected some plants while in the field. Also collected in Ceará anc. Piauí in 1909. Collected with F.Q. Lima.

LOUREIRO, A .

\section{AMAZONAS}

1965

1965 Amazonas; Manaus (116 collections)

Without collector's number but with INPA herbarium number; deposited at INPA. 
LOURTEIG, ALICIA (1911 -

June 1966

PARÁ; Belém \& vicinity

$P$

Small collection made while attending a symposium in Belém. Also collected in Santa Catarina, French Guiana etc.

LUETZELBURG, PHILLIPP FREIHERR VON $(1880$ - 1948)

AMAZONAS; TERR, RORAIMA; TERR. AMAPÁ

1928 - 1929

1928 - 1929 Terr. Amapá; Rio Oiapoque through the Guianas to: Terr. Roraima; Rio Takutu, Rio Uraricoeira, Mount Roraima, Rio Branco: Amazonas; Rin Branco, Rio Negro, Rio Uaupés to Colombia.

Luetzelburg's collecting in Amazonia was as a member of a frontier expedition organized by Marshal Rondon. He also collectea' extensively in northeastern Brazil.

ca 2000 numbers from Amazonia

$M, R$. In addition many Luetzelburg collections were sent to Berlin and subsequently destroyed during World War II, this includes the holotypes of a new species described by Suessenguth and by Pilger. Other duplicates of Luetzelburg collections have been widely distributed by M. e.g. to BRSL,F, NY,W .

For obituary see Suessenguth in Ber Deutsch. Bot. Ges. Jahrg. 68 (Nachrufe) : 65-69. 1955.

LUIZ -- Coêlho, Luiz. F.

MAGUIRE, BASSETT (1904 -

Amazonas; Terr. roraima; ParÁ; Terr. amaPÁ; TERR. RONDÔNIA; MATO GROSSO

\section{Amazonas}

Oct. 1965 Rio Negro; Barcelos, Tapuruquara (60032-60063)

Oct. 1965 Rio Cauabarí to Serra Neblina (60066-60547)
TERR. RORAIMA

Nov. 1954 Serra Tepequem (Nos. 4000040179)

Dec. 1954 Rio Cotinga, Vista Geral (Nos. 40180-40371)

Dec. 1954 Vista Geral (Nos. 40372-40472)

Jan. 1955 Vista Geral (Nos. 40473-40485)

Jan. 1955 Boa Vista (40486)

PARÁ

Aug. 1963 Belém-Brasília highway (5600156073 )

TERR. AMAPÁ

July 1960 Santana, Porto Platon, Rio Oiapoque, Clevelândia (Nos. 47000-47132).

MARANHÃO

Aug. 1963 Belém-Brasília highway (5607456083 .

TERR. RONDÔNIA

Sept. 1963 Vilhena, Pôrto Velho, Madeira-Mamore railroad to Abunã (56537-56802)

MAto GROSSO

Aug.-Sept. 1963 Road Brasília to Pôrto Velho :

Alto Araguaia, Cuiabá, Diamantino, Rio Juruena, Vilhena (56737-56614, 56803-56969) .

NY,IAN,MG,F,GH,K,P,S,U,US etc .

MALME, GUSTAV OSKAR ANDERSSON

(1864 - 1937)

MATO GROSSO

Nov. 1893 - Aug. 1894 Mato Grosso; Cuiabá; Santo Antonio, Serra da Chapada, Corumbá.

June-Dec. 1902; Mar.-June 1903 Mato Grosso ; Corumbá, Cuiabá.

His itinerary never took him into the region of the true Amazonian forest. Until 1891 Malme was known as G.O. Andersson. For further details of Malme's itinerary see Urban (1906) .

S,UPS,LD,R 
MARLIER, G.

\section{Amazonas}

1964

Jan. 1964 Amazonas; Manaus (19 collections)

Collections with INPA herbarium numbers only; deposited at INPA.

\section{MARTINS, OSCAR}

PARÁ

$1906-1916$

Collections at MG

Collector for Museu Goeldi, Belém who worked largely with ornithological collections but who also collected plants in Pará especially around Monte Alegre. Collected mainly 1906 - 1916.

MARTIUS, KARL FRIEDRICH PHILIPP VON (1794 - 1868)

\section{AmazonaS; Pará; Maranhão}

$1818-1820$

Sept. 1810 - Mar. 1820 Amazonas; Parintins, Ilha Tupinambarama, Manaus, Rio Solimốes, Manacapuru, Praia de Pratari. Praia de Goajuratuva, Lago Anury, Coarí, Praia do Sorubims, Uaratapera. Tefé, Nogueira, Rio Japurá, São João do Principe, Vila Bittencourt into Colombia (Rio Apaporis), return via Rin Japurá, Tefé, Manaus.

Mar. - Apr. 1820 Pará; Óbidos, Santarém, Belém

July - Sept. 1819 Pará; Belém \& vicinity, Ric Guamá, Ilha das Onças, Rio Mojú, Rio Jacari, Breves, Rio dos Macacos, Rio Jaburú, Antonio de Gurupá, Pôrto de Móz, Rio Uruará, Santarém, óbidos, Tapera.

June 1818 Maranhão; Rio Parnaíba to Rio Itapecuru, São Luíz.

M,BR,BM,LE,W

Martius travelled with J.B. von Spix, a zoologist.

For further details see Urban (1906).

\section{MATTOS, JOÃO RODRIGUES}

TERR. AMAPÁ

1962

March 1962 Terr. Amapá; Serra do Navic, Pôrto Santana, Pôrto Platon

About 400 numbers

With Nilga Fischer Mattos

SP (duplicates not yet distributed)

\section{MELLO, FRANCISCO}

Amazonas

$1955-1966$

1955 Amazonas; Manaus (319 collections)

1956 Amazonas; Manaus (185 collections)

1962 Amazonas; Careiro, Cambixe (20 collections)

1966 Amazonas; Autaz-Mirim (55 collections)

Earlier collections are without collector's number but have numbers of INPA herbarium. The 1966 collections are Mello numbers 1-55.

MELLO FILHO - Emygdio, L.

MEXIA, YNES (1870 - 1938)

Amazonas; PARÁ

1931

June-Sept. 1931 Amazonas; Rio Amazonas Rio Solimões on to Peru (Dept. Loreto) .

Sept. 1931 Pará; Belém, Acará, Jurutí

Specimens deposited at A,B,BM,C,CAS,F, G,GH,LA,LCU,MICH,MO,NY,P,PH,S,POM, UC, US,USFS,U.

See Mexia, Y, 3000 miles up the Amazon, Bull. Sierra Club 1933.

Mexia also collected in southern Brazil, especially Minas Gerais.

\section{MIRANDA, VICENTE CHERMONT DE}

PARÁ

$1902-1906$

PARÁ; Ilha do Marajó, Belém

MG

Miranda was a farmer on the Ilha do Marajó who collected some plants for the Museu Goeldi. 
MONTEIRO, OSMARINO P.

\section{AMAZONAS}

1967 - 1970

Amazonas; Manaus

Collections without collector's number but with INPA herbarium numbers; deposited in INPA. Also his own numbers 1-25, collecter in 1969.

Also collected widely with other collectors of INPA. His collections were distributed un. der his first name, Osmarino.

MONTEIRO DA COSTA, RAYMONDO C.

PARÁ

1932

PARÁ; lower Rio Tapajós region at Fordlândia, Boa Vista.

F,IAN .

Collected at Ford rubber plantation from trees being felled during clearing operations for rubber tree planting. Monteiro da Costa collected wood samples with many of his specimens. His collecting was arranged by Dr. B.E. Dahlgren of Chicago.

MOORE, HAROLD EMERY JR. (1917 -

\section{Amazonas}

1967

March 1967 Amazonas; Vicinity of Manaus

Collection of Palmae only; about 50 numbers.

INPA,BH,NY lho, L.

With Prance, G. T.; Forero, E. \& Coê-

\section{MOORE, SPENCER LE MARCHANT}

MATO GROSSO

1891

Sept.-Dec, 1891 Mato Grosso; Corumbá, Cuiabá, Santa Anna da Chapada, Serra Arará, Jaugada, Rio Jacoara, Barra do Bugres, Diamantino, Palmera, S. Luis de Cáceres, Corumbá.

$\mathrm{BM}, \mathrm{K}, \mathrm{E}, \mathrm{NY}, \mathrm{P}, \mathrm{R}$
Moore never reached the region of true Amazonian forest. For further details see Moore, S .Le M, Phanerogamic Botany of the Mato Grosso Expedition 1891-92. Trans. Linn. Soc. Lond. II. 6: 265-516. 1895.

MOSES, T.

MARANHÃo

1932, 1938

MARANHÃo; Rio Maracaçumé \& vicinity $\mathrm{BM}$

MOSS, Rev. A. MILES

PARÁ

1930

PARÁ; Belém \& vicinity US 33 numbers.

MOURA, J. G.

\section{MARANHÃO}

1967

Oct.1967 Maranhão; Estação Experimental Florestal, Alto Turi (22 collections) Without collector's number but with INPA herbarium number; deposited at INPA.

\section{MUSEU GOELDI, BELÉM, PARÁ (MG)}

Some earlier material collected by personnel of the Museu Goeldi does not bear the collector's name and must be cited solely as a Museum Collection. These collections are nearly all from Pará especially in the vicinity of Belém.

\section{OLIVEIRA, MANOEL DE}

Amazonas; PARÁ; TERr. Amapá; Maranhã́; GoIÁs

$1958-1970$

AMAZONAS

1958 Rio Apara Maués

52-133

Oct. 1962 Rio Negro, Uaupés 2129-2308

Sept.-Oct. 1963 Manaus-Itacoatiara highway 2658-2845; 2846-3068 
PARÁ

1958 Lago Preto Juruti 2-51

Nov.-Dec. 1959 BR-14 Belém-Brasília highwav 134-857

July 1960 BR-14 Belém-Brasília highway 858-1029

Sept.-Nov. 1960 Belém \& vicinity 1141-1147

Nov. 1960 BR-14 Belém-Brasília highway .... 1148-1150

Dec. 1960 Rio Tocantins, Região de Tucuruí 1151-1262

Feb.-Mar. 1961 Rio Tocantins, Região de Igarapé Miri \& Cametá 1263-1359 A

Apr. 1961 Rio Araguaia, Regiōes de Aragua. tins \& Xambioã 1360-1578 B

Sept. 1961 Belém 1835-1840

May 1962 Northeast Pará; Tiriós 1841-2004

May 1962 Belém 2005-2006

1962 Rio Anabini, tributary of Atúa, Muaná, Marajó 2007-2124

Aug. 1962 Rio Piriá 2125-2127

Oct.-Nov. 1962 Rio Tapajós, Belterra 2309-2312 Rio Tapajós, Fordlândia 2313-2361

Dec. 1962 Belém 2362-2374

Aug. 1963 - Rio Caracurú, tributary of Jari, Igarapé do Inferno ,Rio Caracurú; Logarejo São Miguel, Região de Almeirim 2454-2559

Sept. 1963 - Belém 2560-2578

Mocambo, grounds of IPEAN 2579-2657

1965 Rio Genipapo, Soure 3364-3466

Jan. 1966 - Belém; Bosque Rodrigues Alves 3467-3493

Nov. 1969-Feb. 1970 - Soure Muaná, Marajó 4889-5185

Mar. 1970 - Belém \& vicinity 5186-5202

May-July 1970 - Belém, Ilha do Outeiro ... 5203-5211

1970 - BR-14 Belém-Brasília highway .... 5212-5241

T'ERR. AMAPÁ

Rio Jari, Cachoeira de Sto. Antonio 2438-2453

PARÁ \& TERR, AMAPÁ

1967-1968-1969 - Rio Jari on both the Pará \& Amapá sides 3494-4888
MARANHÃo

July-Sept. 1960 - BR-14 Belém-Brasília highway $1030-1140$

Gorás

Aug. 1961 - Rio Araguáia - Rio Piranha do Araguáia, Logarejo Araguatins 15791834

No date

Pará; Campina Martins Pinheiro, Matapiquara, Bragança Railroad 3069-3363

IAN, NY

OLIVEIRA, J.

TERR, RONDÔNIA

1963

Jan. 1963 Terr. Rondônia; Pôrto Velho

15 collections without collector's number but with INPA herbarium numbers; collected with L. Coêlho \& deposited at INPA.

ONISHI, EUNICE; see Royal Society

OSMARINO - Monteiro, Osmarino P.

PENA, BENTO DA SILVA.

PARÁ

Collector of the Instituto Agronomico, Belém (IAN). He generally collected with other botanists and other collectors but a few collections from around Belém, Pará, were, collected in his own number series.

\section{PEREIRA, AGAPITO}

\section{AMazonas}

1955

Mar. 1955 Amazonas; Manacapuru

4 collections without number deposited at INPA.

\section{PEREIRA, EDMUNDO}

PARÁ; TERR. AMAPÁ

1955, 1957

PARÁ

Sept. 1955 - Pará; Serra do Cachimbo Oct. 1957 - Pará; Belém \& vicinity 
TERr. AMAPÁ

Oct. 1957 - Pôrto Grande, Macapá

\section{$\mathrm{RB}$}

Pereira made two brief collecting visits to Amazonia and made only a small collection. He also collected widely in eastern central Brazil.

PESSOAL DO C.P.F.

(C.P.F. - Centro de Pesquisas Florestais)

\section{AMAZONAS}

1958

1958 Amazonas; Manaus (249 collections)

These sheets bear no collector's names but were collected by personnel of INPA, Manaus. Collections deposited at INPA and widely distributed (MG,NY) .

Many of these collections have the locality as B.A.M. which signifies Brazil, Amazonas, Manaus.

\section{PHILCOX, DAVID}

AMAZONAS; MATO GROSSO

1968

Amazonas

May-June 1968 - Manaus \& vicinity (Nos. 5044-5066)

$\mathrm{K}, \mathrm{INPA}, \mathrm{NY}$

Philcox also collected with G.T. Prance in Amazonas (Rio Urubú) and Terr. Rondônia (Jaciparaná \& Mutumparaná) June-July ... 1968 , and he was also a member of the Royal Society Expedition to Mato Grosso in 19671968.

PILGER, ROBERT (1876 - 1953)

MATO GROSSIO

1898

Feb.-Oct. 1898 - Mato Grosso; Rio São Lourenço, Rio Cuiabá, Serra das Pedras, Rio Cuiabazinho, Rio Parantinga, Rio Ronu- ro, Rio Jatobá, Rio Kuliseiu, Cuiabá .

About 700 numbers, collections lost at Berlin.
PIRES, JOÃO MURÇA

Amazonas; PARÁ; Terr. AMapÁ; Maranhão

1946

Amazonas

Nov. 1946 Parintins 21-25

Ma.ués 26-184;202

March 1947 Manaus 205;208-214

Rio Negro, Macará 227-231

Rio Negro, Tapuruquara 232-238; 1919

Bacia clo alto Rio Negro 1897, 1900-1901

Rio Uaupés, Taraquá 1898-1899

Rio Uaupés, Serra dos Tucanos 1902

Rio Uaupés, 1903-1909; 1913-1918

Rio Içana, Tunuí 1910-1912

April 1947 Rio Negro, Macará 206-207;215-224; 260-262

Rio Negro, Airão 239-241

April 1947 Rio Negro, Tapuruquara 244-259; 263-316; 320-321

Rio Negro, Jucabí 319 \& 322; 324-325; 327 ; 330-331; 336

Rio Negro, Serra do Cabarí 323

Rio Negro, Tatú 355-356

Rio Negro, Marabitanas 468-493

Manaus 225-226

Rio Uaupés, São Gabriel 326, 328-329;

332-335; 337-339

Rio Uaupés 341-354

Uaupés 363-364; 366; 494-550

Ilha das Flores, mouth of Rio Uaupes $340 ; 367-433$

Camanaus 357-362; 365

Vila Içana 434-467

May 1947 Barcelos 551-595; 595-599

Uaupés 600-608

Sept.-Nov. 1947

between Rio Negro \& Barcelos 641-658

Rio Negro, Vila Içana 690

Rio Negro, Foz do Uaupés 1148-1152

Rio Negro, Uanari 1153-1158; 1165-1171

Rio Negro, Vila Alegre 1172-1175

Rio Negro, Tapuruquara 1176-1180

Uaupés $659-682$; 768-780; 814-828

between Ilha das Flores \& Uaupés 767

Rio Uaupés 847-1010; 1025-1147

Rio Uaupés, Serra do Uapici 1159-1164

Ilha das Flores, foz do Uaupés 683-686

near Foz do Uaupés 831-846

Rio Içana, Castanheiro 687-689 
Sept.Nov. 1947 Rio Içana, Maçarico 691-692

Rio Içana, Tunuí 693-697; 704-766;

781-784

Rio Içana, Tatupeua 702-703

Uanarí 785-813; 829-830

Rio Tiquié 1011-1120

Taracuá 1021-1024

Manaus, Estrada de Aleixo 1181-18

Sept.-Nov. Parantins 1277-1280

Maués 1281-1285

Tefé 1286-1362; 1374-1388; 1411-1477

Jan. 1949 Tefé 1480

1959 upper Rio Negro, Merces 7165

Rio Uaupés, Taraquá 7166; 7170-7171; 7442-7616

PARÁ

Sept. 1946 Belém 1-10; 12-20

São Miguel do Guamá 11

Nov. 1946 Tracuateua 185-195

Belém 196-201

Feb. 1947 Belém 204

Apr. 1947 Belém 609-640

Aug. 1947 Ilha, do Marajó 1197-1273

1947 Curralinho 1920-1923

Rio Tocantins, Marabá 1924

March 1948 Belém 1183-1189; 1191-1196

João Coêlho 1190

Aug. 1948 Belém 1274-1276

Nov. 1948 Monte Alegre 1363-1373

Vigia $1389-1410$

Jan. 1949 Belém 1478-1479; 1481-1488; 1490-1494

July 1949 Belém 1526-1527

Aug. 1949 Belém 1784a-1786a

Bacia do alto Rio Tocantins 1929-193e

Oct. 1949 Belém 1796

Nov.-Dec. 1949 Belém 1508; 1834-1861

Santarém 1797-1833

Feb. 1950 Belém 1862; 1863-1883

Peixe-Boi 1884

March 1950 Guamá 1885

Belém 1886-1895

Ilha do Marajó 1896

May 1950 Belém 1937-1938

Rio Tocantins, Marabá 1939

June 1950 Belém 2603

Aug.-Oct. 1950 Belém 2621-2652
Nov.-Dec. 1950 Cacual Grande 2655-2655b

Fordlândia 2653-2654

Belém 2656-2699

April 1951 Belém 3100-3214

Nov. 1951 Belém 3452-3462

Dec. 1951 Belterra, Pindobal 3463-3475

Cacual Grande $3476-3477$; $3478-3480$

Belterra 3481-3499

Carocal 3500-3507

Rio Tapajós, Aveiros 3508-3509

Rio Tapajós, Caroçal 3510-3523

Rio Tapajós, Vila-Nova 3524-3638

Dec. 1951 Rio Tapajós, Boa. Esperança 3639-3652

Rio Tapajós, São Francisco 3653-3654

Rio Tapajós, Santa Maria 3658-3659

Flexal 3655-3657

Barra do Rio Juruena 3660-3679

Vila. Nova $3680-3692$

Rio Juruena Falls - also in Mato Grosso, Amazonas 3693-3731

Jan. 1952 Rio São Manoel, Bom Jardim 3732-3737

Rio São Manoel, also in Mato Grosso.

Amazonas 3750-3768

Barra dio Rio São Manoel 3792-3802:

3803-3880; 3881-3900; 3925-3934

Conceição 3901-3924

Rio Tapajós, between Barra \& Vila No. va $3935-3942$

Vila Nova $3943-4041$

Jacareacanga airport 4042-4052

Feb. 1952 Rio Tapajós, Itauituba 40534060

March 1952 Belém 4062-4064; 4066-4071

May 1952 Rio Aruá 4191

Cachoeira do Rio Aruá 4197-4221

Rio Aruá waterfall 4241-4242

Santarém 4243-4249

June 1952 Vigia 4073-4093

Rio Arapiuns 4192-4196; 4224-4240

Rio Maró 4222-4223

Maicurú 4250-4324

Juiy 1952 Belém $4184 a-4187$

Rio Mapuera, 4188-4190

August 1952 Castanhal, Colônia 3 de Outubro $4094-4184$

Sept. 1952 Be'ém 4325

Marituba 4326 
7203-7221; 7259; 7265; 7617-7635; 76377643

Rio Arapiuns 4347-4383; 4389-4404

Rio Maró 4384-4388

Dec. 1952 Belém 4405-4440

Jan. 1953 Castanhal 4441-4457

Feb. 1953 Belém 4458-4465

March 1953 Belém 4469-4473

May 1953 Belém 4474-4499

Ourém 4500-4514

July 1953 Belém 4515-4520

Rio Guamá 4586-4640

Aug.-Nov. 1953 Cametá 4521-4533

Belém 4534-4552

Dec. 1953 Rio Mojú 4569-4585

Jan. 1954 Belém 4641-4660

March 1954 Monte Alegre 4568

Belém 4568a

Apr. 1954 Bragança 4818-4856

May-June 1954 Castanhal 4661-4663

Belém 4664-4669; 4685-4686

Belém, Guajará campina 4670-4684

Aug. 1954 Gurupá 4690-4741

Nov. 1954 Belém 4742-4755

Dec. 1954 Vigia 4756

Belém 4553-4554; 4557-4567

Apr. 1955 Castanhal 4857-4858

July 1956 Breves 4860-6030

Sept. 1956 Belém 4859

Oct. 1956 Belém 6031-6033

Vigia 6034-6050

Nov. 1956 Belém 6051-6052

Dec. 1956 Serra do Cachimbo 6062-6476; 6549-6556

Santarém 6477-6525

Maicurú 6526-6548

Belém 6557-6558

Feb. 1957 stream on Rio Maicurú 6559-6572

May 1957 Belém 6572 a -6573a ; 6574-6575; 6614

Oct. 1957 Breves 6615-6692

March 1958 Belém 6694-6742

May 1958 Vigia $6743-6758$

June 1958 Belém 6759-6760b; 6838-6849

Municipality of Marapanim 6761-6784

Belém, grounds of Pirelli Co. 6785-6837;

- $6850-7144$

Dec. 1958 Santarém $7145-7151$

1959 Belém 7152-7164; 7167-7169; 7173-7178;
7203-7221; 7259; 7265; 7617-7635;

7637-7643

Curuauna 7179-7199a

Vigia 7200

Marapanim 7201-7202

Santarém 7222-7258; 7260-7264; 72667441

Belém-Brasília Highway $\mathrm{Km} 93$ 76367648

Belém-Brasília Highway Km 87 76447646

Belém-Brasília Highway Km 97 76497654

1960 Belém 7655-7670a; 7672-7676; 7679 7685; 7796-7823

1960 Belém, grounds of Pirelli Co. 7671 Santarém 7678

1961

June \& July Belém, grounds of Pirelli C. 7824 Belém 7825-7843; 7847-7850

Nov. 1962 Belém 48894-48906

Amapá

March 1952 Macapá 4065

1955 Mazagão 4757-4764

Feb. 1955 Macapá 4765-4769; 4789-4796

Curiau 4770-4771; 4806-4817

Pôrto Platon 4772-4784

Mazagão 4785

Cupichi 4786-4788

Matapi 4797-4805

Nov. 1956 Pôrto Platon 6053-6061

1960 Clevelândia 7686-7749; 7792-7793

Rio Oiapoque 7750-7791; 7794-7795

Aug.-Oct. 1960 Rio Oiapoque; Clevelândia $47372-47373 ; 47459-47466$; 47533-47538; 48557-48580; 48810-48893

July-Oct. 1961 Rio Araguari, Serra do Navio $50250-51663$

(with W. A. Rodrigues \& G. C. Irvine)

July-Aug. 1962 Coastal region of Amapá 51970-52688

(with P.B. Cavalcante)

MARANHÃO

Feb. 1947 Carolina 203

April 1949 São Luíz 1509-1513

July 1949 Guimarães 1514-1525

May 1950 Carolina 1940-2530 
WITHOUT LOCALITY

1947 1925-1927

Collections at IAN, duplicates widely disiributed NY,UB,US

The high numbers in 1960-62 were collected in the New York Botanical Garden number series.

POEPPIG, EDUARD FRIEDRICH (1798-1868)

AMAZONAS; PARÁ

$1831-1832$

July 1831 - Apr. 1832-Amazonas; Tabatinga to Tefé

Pará; Belém, mouth of Rio Tocantins.

W,LE,BM,BR,G,GOET,LZ

For further information see Peoppig, E.F., Reise in Chile, Peru und auf dem Amazonenstrom Während der Jahr 1827-32. Leipzig 1835-36, and also Urban (1906) .

POHL, JOHANN EMANUEL (1782-1834)

$1817-1821$

Pohl collected in Brazil $1817-1821$. He travelled extensively in southern Brazil but only just touched the fringes of Amazonia in Mato Grosso, for full details of his itinerary see Urban (1906).

PRANCE, GHILLEAN TOLMIE (1937-

Amazonas; TrRr. Roraima; PARÁ; Maranhão; ACRE; TERR. RONDÔNIA; MATO GROSSO 1965 -

\section{GMAZONAS}

Aug.-Nov. 1965 Manaus 2079-2081

Aug. 1966-Mar. 1967 Manaus 2114-2282; 2596-2724; 3020-3202; 3613-3944; 46254803

Sept.-Dec. 1966 Rio Purus, Bôca do Acre; Rio Madeira, Humaitá 2283-2595; 32033612

May-June 1968 Rio Urubú 4861-5151 (with D. Philcox \& W.A. Rodrigues)

Oct.-Nov. 1968 Rio Purus, Lábrea 7970-8225

Sept.-Oct. 1968 Rio Iaco; 7889-7926 (with D. Coêlho)
Feb.-Mar. 1969 Tototobi 10196-10412 (with J.R. Steward)

May 1968-Mar. 1969 Manaus 4824-4860; 7193-7227; 9014-9095; 9098-9099; 10413-10456

TERR, RORAIMA

Jan.-Feb. 1967 Rio Mucajaí, Rio Apiaú 39454236 (with E. Forero)

Rio Uraricoera, Serra do Tepequem 4237-4612 (with E. Forero) Boa Vista 4613-4624 (with E. Forero)

Jan.-Mar. 1969 Boa Vista \& vicinity 9100. 9160; 9504-9582 (with J.R. Steward) Serra da Lua 9161-9503 (with J. R. Steward)

Auaris 9583-9869 (with J.R. Steward) Serra dos Surucucus 9870-10195 (with J.R. Steward)

PARÁ

July-Aug. 1964 Belém-Brasília highway, Gurupí, Paragominas, Capanema, Rio Piriá, Cachoeira, Guamá, Gurupí 5843158993 (with N.T. da Silva)

Aug-Nov. 1965 Rio Pacajá, Portel 1255-1667 (with T.D. Pennington \& N.T. da Silva) Belém to Rio Gurupí (road BR-22) 1668-2078 (with T. D. Pennington).

MARANHÃO

July-Aug. 1964 Carolina, Estreita, Imperatriz, \& northern Goiás 58505-58666, 58971-58993 (with N.T. da Silva)

ACRE

Sept.-Dec. 1966 Rio Juruá, Cruzeiro do Sul 2725-3019

Sept.-Oct. 1968 Tarauacá, Sena Madureira 7228-7969 (with D. Coêlho)

TERR. RONDÔNIA

June-Aug., Nov.-Dec. 1968 Jaciparaná; Abunã, Ribeirão, Guajará-Mirim, Sta. Bárbara, Pôrto Velho, 5152-5692; 57335765 ; 5808-5843; 5874-6053; 6065-6086; $6161-6200$; 6232-6242; 6256-6260; 62786299 ; 6432-6465; 6505-6529; 6543-6592; 6617-7019; 7156-7192 (with L. Coêlho, E. Forero, D. Philcox \& B.L. Wrigley); 
$8226-8350 ; 8451-8510 ; 8539-8559 ; 8573-$ 8637 ; 8684-8702; 8709-8727; 8759-9013 (with W.A. Rodrigues).

The missing numbers in the Rondônia series were collected in Bolivia, State of Pando : 5693-5732; 5766-5807; 5845. 5873 ; 6054-6064; 6087-6160; 6201-6231; $6243-6255 ; 6261-6277$; 6466-6504; 65306542; 6593-6616; 8351-8450; 8511-8538; $8560-8572$; 8638-8683; 8703-8708; 87288758 .

\section{MATO GROSSO}

Sept.-Oct. 1964 Barro de Garças, Xavantina, Garapú, Rio 7 de Setembro 59088-59449 (with N.T. da Silva)

\section{INPA,NY,F,GH,K,IAN,MG,R,S,U,US}

The 1964 collection was collected under the New York Botanical Garden number series. The remainder under his own number series. For the collections between 1965 \& 1969 he was assisted by José F. Ramos.

RAMOS, JOSÉ F.; see Prance, G.T. \& Royal Society

RATTER, J.A.; see Royal Society

REYNOLDS, DON R.

Amazonas, PARá,

May-June 1969

AMAzonas; Manaus and vicinity

PARÁ; Belém and vicinity

Mycological collections only, deposited at Cornell University.

\section{RICHARD, LOUIS CLAUDE MARIE}

(1754 - 1821)

PARá

1785

PARÁ; vicinity of Belém

$$
\text { P,C }
$$

Richard also collected in French Guiana and the Antilles.

\section{RICHARDS, PAUL WESTMACOTT}

AMAZONAS; MATO GROSSO

1968

AMAZONAS

Nov. 1968 - Manaus \& vicinity

In Amazonas Richards collected unäer the W.A. Rodrigues number series and made a few collections only. He was accompanied by G. C. G. Argent. He also collected extensively in Mato Grosso with the Royal Society expedition.

RIEDEL, LUDWIG (1790 - 1861)

Amazonas; PARÁ; TERr. RONDÔNIA; M. grosso 1829

Ámazonas; Rio Madeira, Borba, Manaus, Rio Amazonas

Pará; Óbidos, Santarém, Belém

TERr. rôndonia; Rio Guaporé, Rio Madeira

Mato Grosso; Cuiabá, Serra da Chapada, Ric Guaporé, Cascalvasco.

1600 numbers some from south of Ama. zonia .

\section{$\mathrm{LE}, \mathrm{R}, \mathrm{K}, \mathrm{BR}, \mathrm{G}, \mathrm{NY}, \mathrm{US}, \mathrm{Z}$}

In addition to the above collection Riedel collected widely in southern Brazil.

For full details see Urban (1906).

RODRIGUES, JOÃO BARBOSA (1842-1909)

Amazonas; Pará; Maranhão

1873 - 1874 Amazonas; Rio Uatumá, Rio Jatapu, Rio Urubú (Partly with J.W.H. Traill)

1884 - 1885 Amazonas; Rio Iauaperi, Mahuá, Rio Negro, Rio Purus

1889 Amazonas; Rio Jauari, Rio Solimões.

1871 - 1872 Pará; Rio Capim, Rio Tocantins, Santarém, Rio Tapajós, Rio Xingú, Rio Trambetas; Amazonas, Serra Parintins. $\mathrm{RB}, \mathrm{R}$

The herbarium collections of Rodrigues at $\mathrm{RB} \& \mathrm{R}$ are quite small and incomplete, and often it is necessary to typify his species from plates in his publications. 
The collection which he made during his 7 years as director of the Museu Botânico do Amazonas from 1884-1890 were apparently completely lost since no trace of them can be found in Manaus or anywhere else. He left them in Manaus, and since the museum ceased to exist on his return to Rio de Janeiro the specimens were not cared for and probably eventually thrown away.

\section{RODRIGUES, JOSÉ S.}

AMAZONAS

1959

AMAZONAS

Feb.-Mar. 1959 - Upper Rio Negro, Uaupés, Illha das Flores, Rio Uaupés, Taraquá.

\section{IAN, NY}

Collected in his own number series, about 160 numbers, accompanied by J.M. Pires \& N.T. Silva.

\section{RODRIGUES, RODOLFO SIQUEIRA}

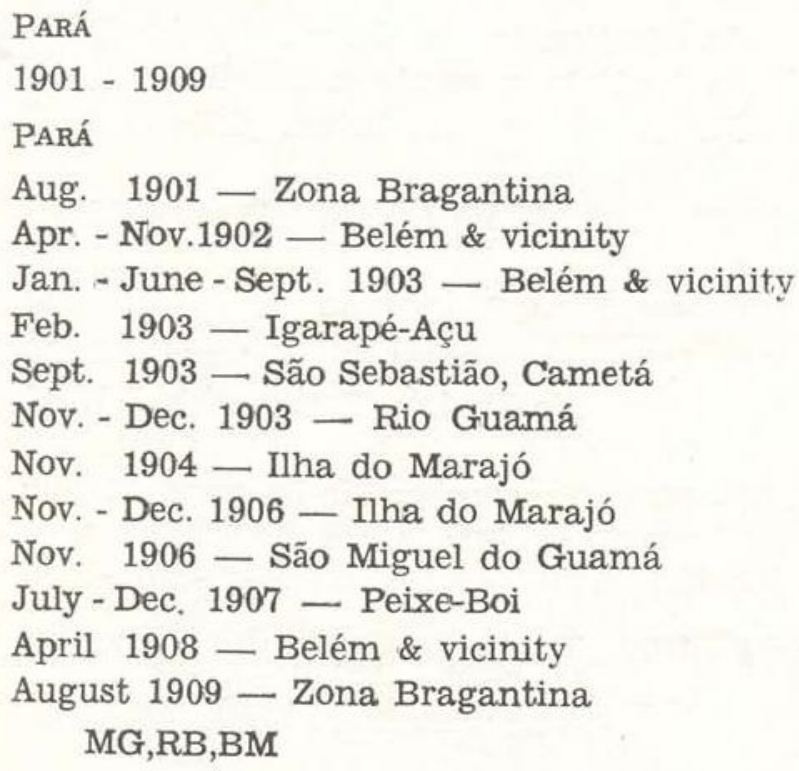

Collector \& Administrator at the Museu Goeldi whosễ plants were numbered in the Museu mseries. Many of his collections are labelled R. Siqueira.

\section{RODRIGUES, WILLIAM ANTONIO}

AMAZONAS; TERr. RORAIMA; PARÁ; TERr. AMAPÁ; MARANHÃO; TERR. RONDÔNIA

1954

\section{AMAZONAS}

1954

Oct. - Dec. Manaus 2 Collections

1955 Manaus 73 specimens without number

1956

Nov. Rio Uatumã 201-261

Silves $262-279$

Dec. Rio Urubú 280-356

1957

Jan. Lower Rio Negro, Tarumãzinho 357-358

Apr. Rio Manacaparu 370-455

Manaus 456-605

1958

Manaus 606 ; 626-655; 710; 761-778; 8 without number

March Cucuí 608-615

Apr. Rio Iça - Pelotão Fronteira Ipiranga 616-619

Rio Japurá Pelotão Fronteira Japurả 620-624

Tabatinga Pelotão Fronteira without number

Nov. Manaus (with A. Aubréville) 779-785 \& $990 ; 1288-1299$

Dec. Between Manaus \& Bôca do Rio Apuaú 711-760

1959

Apr. Manaus 982-986

Manaus (with J. Chagas) 991-998;

$1170-1183 ; 1195-1201 ; 1204-1212 ; 1229$ $1236 ; 1239-1245$.

Feb. 1959 Uaupés, Ilha das Flores 999-1114 Taracuá 997; 1115-1169

Manaus (with L. Coêlho) 1184-1194, 1246-1949

1959 Manaus (with D. Coêho) 1237-1238 \& 1950

Manaus 1184-1201; 1237-1287; 13001387; 1449-1453

Nov. Tefé, Maraã (with L. Coêlho) 1388-1448

1960 Manaus 1455-1462; 1470-1552; 1629; 1946-1653; 1664-2040. 
Jan. Uaupés (with L. Coêlho) 1463-1469

March Nova Olinda, 1553-1555

Rio Acarí, Borba 1556-1605

April Rio Canumã, Borba 1606-1624

Borba, Paranã Araria 1625-1628

Careiro (with D. Coêlho) 1630-1645

May Tefé, Vila Caambé (with L. Coêlho) 1653-1663

1961 Manaus 2040-2057; 2065-2070; $2077-$ 2079; 2082-2368; 2386-2608; 2665-3654; 3829-3902; 3929-3953; 3971-3975; 4041-4146.

Jan. Tefé 2058-2064

Rio Cueiras 2080-2081

Feb. Manaus (with Hiko-Ishi-Oka) 2071-2076

April Careiro, Cambixe (with F. Mello) 2369-2385

Dec. Rio Parauari, Maúes (with L. Coêlhn) 3903-3928

Rio Cueiras (with Byron \& D. Coêlho) 3954-3970; 3976-4040

1962 Manaus 4047-4147; 4158-4220; 4334$4343 ; 4357-4420 ; 4422-4741 ; 4774-4851$; 4883-4894; 4905-4947.

Oct. - Nov.Uaupés (with D. Coêlho) 4742-4751

Oct. - Nov. Içana (with D. Coêlho) 4752-4754a

Cucuí (with D. Coêlho) 4555-4763

Upper Rio Negro, Bôca do Rio Xie (with D. Coêlho) 4764-4773

Lower Rio Negro, Bôca do Igarapé Araras (with D. Coêlho) 4852-4860

Rio Cueiras (with D. Coêlho) 4861-4882; 4895-4904

1963 Manaus 4948-4968; 4977-5063; 51855188; 5191-5341; 5364-5518; 5524-5651; 7518-7530

Feb. Careiro (with F. Mello) 4869-4976

July Puraquequara (with F. Mello) 5342-5362

Oct. Igarapé das Araras 5519-5523

1964 Manaus 5652-5777; 5792-5844; 5901$6023 ; 6027-6035 ; 6729-6785 ; 6805-6819$

April Rio Tiririca, tributary of Rio Preto (with D. Coêlho) 5778-5791

May-June Rio Preto (with D. Coêlho)

- 5845-5878

June Careiro, Igarapé Grande 5879-5900

Aug. Rio Cueiras (with D. Coêlho) 6024-602
Sept. Rio Cueiras 6036-6099; 6700-6728

Nov. - Dec. Maués (with L. Coêlho) 67866794; 6800-6803

1965 Manaus 6820-7033; 7373-7378

Aug. - Dec. Manaus-Itacoatiara highway 7034-7372; 7407-7514; 7691-7705; 79308106; 8143-8170.

1966 Manaus 7379; 7720-7735; 7803-7929

Apr. Maués, Vila Limão-Limoeiro (with L. Coêlho) 7706-7719

Autaz-Mirim (with F. Mello) 7736-7802

1967 Manaus 8289-8342; 8395

Ma.y Boca do Rio Branco (with L. Coêlho) 8343-8393

Rio Padauari, alto Rio Negro (with L. Coêlho) 8394

1968 Manaus 8396-8562; 8566-8595

Sept. Manaus (with P. Richards) 8563-8565

TERR. RORAIMA

1954

Nov. - Dec. Normandia 1-72 \& 625

Fazenda S. Luíz 73-78

Pedra Branca 79-89

Serra Maturuca 90-113

Rio Ailã 114-119; 175-183

Region between Rios Cana \& Maú

120-174

Cotingo 184-188 - 1 without number

Vista Geral 189-197 — 5 without number

1958

Nov. Boz Vista, Taiano, Foz Florestal (with A. Aubréville) 656-709

1962

Feb. Rio Surumu (with F. Mello) 4344-4356

PARÁ

1959

June Belém 1202

July Vigia, Campina do Palha 1213-1228

1961

March Vigia 2396

1963

April Quatipuru 5064-5184; 5189-5190 
TERr. Amapá

1961

July - Aug. Serra. do Navio 3655-3828; 3953; 4148-4157

MARANHÃo

1966

Sept. Zé Doca 8262-8268

Alto Turi $8253-8261$

TERR, RONDÔNIA

1962

Jan. - Feb. Forte Príncipe do Beira (with Byron \& L. Coêlho) 4221-4333.

First set of specimens deposited at INPA, others widely distributed in Brazil, e.g. MG, IAN,RB, some at NY.

Collections made with a large number of field assistants whose names appear on the labels and are mostly mentioned here under their own names.

\section{ROYAL SOCIETY EXPEDITION}

From 1966-1968 the British Royal Society conducted an extensive expedition in Mato Grosso. Much of the collecting was made south of the region covered by this index. However, important southern range extensions of Amazonian plants were made in the northernmost part of the area covered by the expedition. The expedition's base camp and headquarters were on the road from Xavantina to Cachimbo at $12^{\circ} 51^{\prime}, \mathrm{S} ; 51^{\circ} 52^{\prime}$ 'W. During the expedition many differente collectors visited the region each for a few months. Since all participants collected in the same region the full details are not given under the individuals. Most of them collected in the vicinity of Xavantina, along the road from Xavantina to the expedition's base camp over $200 \mathrm{~km}$. north of Xavantina, in the vicinity of the base camp and up to $60 \mathrm{~km}$. north of it, also around the Rio suiá Missu crossing. Some collectors collected as far south as the Goiás border at Barra do Garças. The participants were as follows :
ARGENT, GEORGE C.G.

Aug. - Nov. 1967; June-Sept. 1968

(Nos. 6297-6758)

Argent collected both in his own number series and in P.W. Richards' number series. Since both series were around the same numbers care should be taken in citation of Argent collections.

BARROSO, GRAZIELA M. Oct. 1968

EITEN, GEORGE \& LIENE Aug.-Oct. 1968

GIFFORD, D.R., June-Sept. 1967; Feb.-Apr. 1968. (Nos. 1-150).

GOLDSMITH, F.B. Mar. - May. (Nos 1-223. L1-23) .

HARLEY, RAYMOND M. Aug. 1968-Jan. 1969 (Nos. $10001-11511$ ).

HUNT, DAVID R.

1966

ONISHI, EUNICE

Cet 1968; Jan. - Feb. 1969. (Nos. 860-1004)

PHILCOX, DAVID

Nov. 1967 - Apr. 1968. (Nos. 3001 - 5103)

RATTER, J.A.

June-Sept. 1967; Mar.-July 1968 (Nos. 1-2155) RICHARDS, PAUL W.

Aug.-.Nov. 1967; June-Sept. 1968 (Nos. 6401-6960)

In addition to the above collectors some of the field assistants made cullections of their own in the number series of one or more of the above collectors. The field assistants whose names appear first on any labels are :

CASTRO, R.A. de

FONSÊCA, SIDNEY

$1967-1968$

RAMOS, JOSÉ, F.

SANTOS, RAIMUNDO R.

SOUZA, RAIMUNDO

The top sets of all the above collections are at $\mathrm{K}$ \& UB; the duplicates will be widely distributed from $\mathrm{K}$, to NY,P,RB,MO,UC,IAN, S,VEN,AAU . 
RUSBY, HENRY HURD (1855 - 1940)

AMAZONAS; PARÁ; TERR. RONDÔNIA

$1884-1885$

From Bolivia to Terr. Rondônia; Rio Madeira

to Amazonas; Rio Madeira, Manaus to Pará, Belém

1921 - 1922

From Bolivia to Terr. Rondônia; Rio Madeita to Amazonas; Manaus

$\mathrm{NY}, \mathrm{B}, \mathrm{BKL}, \mathrm{BM}, \mathrm{E}, \mathrm{G}, \mathrm{GH}, \mathrm{K}, \mathrm{LE}, \mathrm{LA}, \mathrm{MICH}, \mathrm{MO}$, PH, TRIN, US, W

Very few collections during Brazilian part of his expeditions, collected mostly in Bolivian Amazonia. The 1921-1922 trip was as botanist to the Mulford Biological expedition.

SACCO, J.C.; see Fromm, E.

SAMPAIO, ALBERTO JOSÉ DE (1881 -

PARÁ

1928

Sept. - Dec. 1928 Pará; Oriximiná, Rio Cuminá, Serra de Tumucumaque

$\mathrm{R}$

970 numbers (numbered 4964-5930)

For further details see Sampaio, A.J. de, Arch. Mus. Nac. Rio de Janeiro 35. 1933.

SANTOS, E.; see Fromm, E.

SANTOS, R.R.; see Royal Society

SASTRE, CLAUDE

PARÁ; TERr. Amapá

1968

PARÁ

Mar. 1968 Peixe-Boi 45-65

Plantation de Paricatuba, Belém-Mosqueiro road $85-107 ; 130-167$

Rio Guamá, Bocadora 108-113

Rio Ora, Maje de Guamá 114-129

TERR. AMAPÁ

Marçh 1968 Macapá airfield 168-169

Amapá airfield 170-174

Oiapoque airfield 175-177
Collected with his wife France Sastre

Nos. $45-167$ at P,IAN,CAY

168-177 at P,CAY ialists.

Duplicates also widely distributed to spec-

SCHMIDT, HERMAN; see Weiss, L.

SCHOMBURGK, MORITZ RICHARD

(1811 - 1891)

TERR, RORAIMA

1842

Apr. - May Rio Tacutu from Conceição do Maú to about $2^{\circ} \mathrm{N}$ collecting in Guyana and Brazil.

Sept. - Oct. Conceição do Maú, Rio Tacutu, Rio Surumu, Rio Cotinga to Venezuela to Mount Roraima.

Collections widely distributed in Europs $\mathrm{BM}, \mathrm{G}, \mathrm{K}, \mathrm{OXF}, \mathrm{P}$

Collected mainly in Guyana but visited Roraima Territory as well.

For further details see Schomburgk, M.R, Reisen in Britisch-Guiana.

3 vols. 1847 - 1849; Vol. 1 \& 2 translated into English by W.E. Roth, Georgetown 1922, 23.

\section{SCHOMBURGK, ROBERT HERMANN}

(1804 - 1865),

AMAZONAS; TERR, RORAIMA

1839

March Amazonas; Rio Negro from Cucui, Uaupés, Barcelos to Carvoeiro

April Terr. Roraima; Carvoeiro, Rio Branco to Rio Tacutú to Conceição do Maú .

Also accompanied his brother's expedition which went through Roraima Territory 1842. See Richard Schomburgk. Collected mainly in Guyana apart from the one expedition through Brazil.

For further details see :

Robert Hermann Schomburgk's travels in Guiana and on the Orinoco, edited by O.A. Schomburgk, Translated by W.E. Roth, Georgetown 1931. 
SCHULTES, RICHARD EVANS (1915 -

\section{AMAZONAS}

1945; 1947-1948; 1967

August 1945 - Rio Marmelos

Sept. 1947 - 1948 Upper Rio Negro region; Barcelos to Colombian \& Venezuelan frontier; Serra Jacanúm, Rio Uaupés, Cucuí, Rio Denití, Rio Taraira, Rio Marmelos (with F. López approximate Schultes numbers 8700-10350) .

1967 - Manaus \& vicinity (24503-24509; 24594-24623)

Rio Negro, Ilha Gavião mouth of Rio Branco (24510-24540; 24580-24593)

Tapuruquara 24541-24550; 24553

Rio Maraniá 24551-24552

Serra Jacamin 24554

Rio Cauaburi 24555-24579

ECON,GH,NY,IAN,INPA

Schultes also collected much more exten. sive y in Amazonian Colombia.

\section{SCHWACKE, CARL AUGUST WILHELM}

(1848 - 1904)

AMAZONAS; PARÁ; MARANHÃo

1877-78

Maranhão; Pará; Amazonas; Rio Solimões, to Tabatinga

1882

PARÁ; AMAzonas (Manaus)

\section{$\mathrm{R}$, GOET}

1877-78 trip he was accompanied by C. Jobert, and 1882 trip by Netto.

Schwacke collected about 14,000 specimens in Brazil, but the majority were from the south. The numeration of his specimens is confused since some bear his own number series and some a separate number series for each expedition. In addition to this much Schwacke material was pirated by Glaziou. Many collections labelled 'Glaziou, environs of Rio de Janeiro' are in fact Schwacke collections from Amazonia.

The first set of Schwacke collections belonging to one of the Minas Gerais herbaria was lost in transit between Brazil and Italy, but the Museu Nacional, Rio de Janeiro has the largest holdings of his material.

For further details see Urban (1906)
SIEBER, FRIEDRICH WILHELM

PARÁ

1801 - 1807

PARÁ; Belém \& vicinity, Rio Tocantins to $\mathrm{Ca}$ metá, Gurupá, Rio Preto, Monte Aie. gre, Santarém, óbidos.

B (Herb Willdenow), BR,M

The first set of Sieber material was deposited at Berlin, much of this was in the Willcienow herbarium and consequently was not lost in World War II, some for his collecting in the general herbarium of Berlin were lost in the war.

\section{SILVA, ANTONIO DA}

PARÁ

1949

PARÁ; all collections from the vicinity of Belém 1000 numbers

JAN,US,NY

Also collected with W.A. Archer in 1942-1943.

\section{SILVA, JOSÉ FRANCISCO DA}

TERR, RONDÔNIA

1952

1952 - Terr. Rondônia; Pôrto Velho \& vicinity $1-452$

IAN

\section{SILVA, MANOEL}

PARÁ

Apr. - Sept. 1967 Pará; Santarém, Reserva Florestal Curuá-Una. 12 collections without collector's number, with INPA numbers.

\section{SILVA, MILTON GONÇALVES DA}

AMAZONAS; TÉRR, RORAIMA; PARÁ

1964 - 1970

AMAZONAS

1967 - Mar. 23 - Apr. 28; Manaus, Rio Negro, Rio Solimões \& Rio Purus

1968 - Aug. 7 - Oct. 26; Parintins, Rio Negro, Puraquequara, Codajás, Fonte Boa, Sto. Antonio de Içá. 
TERR, RORAIMA

1964 - Feb. 20 - Apr. 9

PARÁ

1964 - Oct. 20; Zona Bragantina, Taciateua

1966 - Jan. 12; Belém, Utinga

Jan. 15-21; Belém-Brasília highway, Paragominas, Rio Uríam, Tomé-Açí; Rio Capim.

Feb. 10; Benevides, road BelémMosqueiro

Feb. 16-17; Marapanim

Feb. 20-23; Acará; Igarapé Jacaréquara, Sítio Tapera

July 9-12; Quatipurú

July 28-3; Curuçá-São Caetano de Odivelas road

Aug. 10-12; Capitão Poço

Oct. 5; Vigia, Campo do Caimbé

1967 - May 27; Vigia, Campo do Palha July 7-9; R. Mojú

1968 - Jan. 10 - Feb. 18; Óbidos, Oriximiná, Faro, Rio Jamundá, Rio Cuminámi rim, Santarém, Rio Maicá, Serra Taperinha, Curuá-Una.

1968 - Aug. - Oct.; Lower Rio Tapajós, Santarém, Gurupá, Xingú, Porto de $\mathrm{Moz}$, Almeirim, Monte Alegre, Ric Tapajós, Alter do Chão, Fordlândia, Oriximiná, road to óbidos .

1969 - Santarém, estrada do Palhão August 10 - Sept. 21. - Nos. 2201 thru 2660

1970 - Marabá, Serra dos Carajás Apr. - Nos. 2661-2676

MG, Nos. 2201-2659 at NY.

SILVA, NILO TOMÁS DA

AMAZONAS; TERr, RORAIMA; PARÁ; MARANhão: TERR, RONDÔNIA

TERR, RORAIMA

Rio Branco 853-890

PARÁ

Pará 116-117

Belém; Bosque Municipal 1-93; 125 135

Belém;, IAN 94-107; 118-124; 136; 192-194; 287-324; 335-345; 473-478; 507-568; $632-644 ; 826-827$
Belém; margin of Rio Guamá 137-138; 265-272

Belém; Mata do Cafezal; margin of Rio Gua. má 191-300

Belém; Rio Guamá; Municipality of Capim 569-580

Belćm; Mata do Mocambo 469-472

Bragança railroad, Santa Izabel 108-115

Vigia; margin of Rio Teixá 139-176

Vigia; s.n.

Campo de Peixe Boi, Bragança railroad $177-190 ; 325-334$

Rio Capim; Igarapé Putiritá 451-467

Breves 468

KM 100 Belém Brasília highway 581-621; 627-631

Pedreirinha, Municipality of Ananindeua 622-626

Municipality of Conceição do Araguaia $645-661 ; 797-820$

Região de Gorotire, Municipality of Altamira 662-796

Região de Gorotire 821-825; 841

Rio Acará 828-839

Belém IPEAN; Cafezal, Reserva Black 842-852.

Região do Rio Jarí; Monte Dourado 891-2894

Estrada do Planalto, Km 13, Estrada de São Miguel, $\mathrm{Km} \mathrm{30,} \mathrm{Estrada} \mathrm{de} \mathrm{Munguba}$ $\mathrm{Km}$ 4, Estrada, entre Planalto e Tin. guelin $\mathrm{Km} \mathrm{11}$, Estrada Tinguelin, $\mathrm{Km}$ $21 \quad 2895-3219$.

TERR, RONDÔNIA

Pôrto Velho 346-450

No Herbarium specimens exist for numbers 196-264.

Silva also made the following collections in the New York Botanical Garden number series.

Nov. 1963 Maranhão; Belém-Brasília road 57774-57793

Nov. 1963 - Feb. 1964 Pará; Belém-Brasília road 57794-57796; 57813-57827; 57855-57857.

Dec. 1963 - May 1964 Pará; Belém 5779757812; 57828-57854; 57858-57890

Mar. 1965 Pará; Belém 59707-59737

Oct. 1965 Amazonas; Rio Negro; Barcelos. Tapuruquara 60032-60063 
1965 Amazonas; Rio Cauaburí to Serre Neblina 60066-60547

1965 - 1966 - Amazonas; Rio Cauabarí to Serra Neblina (with Umbelino Brazão) 60600-61001

IAN,NY

SIQUEIRA - Rodrigues, R. Siqueira

SOTO, ELIAS DE LA

AMAZONAS

1960

Jan. 1960 Amazonas; Manaus

2 collections without numbers deposited at INPA.

\section{SMITH, ALBERT CHARLES (1906 -}

PARÁ

Oct. - Nov. 1929 Collected with E.P Kilip see under Killip

Jan. 1938 Pará; Serra Akaraí (2 days oniy Nos. 2929-3004 from Brazil).

NY,A,Y

regions.

Smith has collected widely in other

SMITH, C.W.

Amazonas; Pará

$1941-1942$

AMAZONAS

Sept. 1941 - Amazonas; Rio Madeira, Rio Manicoré 12214-12226

PARÁ

Apr. 1941 - Pará; Basin of Rio Acapu \& other localities

Mar. 1942 - Pará; Portel Municipality 12501-12522

Apr. 1942 - Pará; Rio Xingú 12580-12589

Smith collected for the Chicle Development Corporation under the supervision oi B.A. Krukoff. His collections were entirely of latex bearing trees, especially Sapotaceae, and were made in the Krukoff number series.

SNETHLAGE, EMIL HEINRICH

PARÁ; TERr. AMAPÁ

1905 - 1919
PARÁ

Cct. 1905 - Santo Antonio do Prata

Dec. 1905 - Marajó

Feb. 1906 - Monte Alegre

Oct. 1906 - Rio Guamá

Dec. 1906 Jan. 1907 - Rio Tapajós, Itaitu. ba, Goyana, Vila Braga

Apr. - May 1907 Rio Tocantins, Alcobaca, Arumatheua

May - June 1908 Peixe-Boi, Quati-Purú

July - Aug. 1907 Monte Alegre, Ereré, Ric Maecurú

Oct. - Dec. 1908 Rio Tapajós, Rio Jamauchin!

May - Oct. 1909 Rio Xingú, Rio Iriri, Ríc Curuá, Rio Jamauchim

Oct. 1910 Santa Isabel

Dec. 1910 Rio Tocantins

June 1911 Ananindeua Belém-Bragança railroad

July 1911 Apehú Belém-Bragança railroad

Aug. 1911 Providência Belém-Bragança railroad

Sept. - Oct. 1911 Rio Tapajós

Jan. - Feb. 1912 óbidos, Rio Jamundá, Faro

Dec. 1912 Arumanduba

Aug. 1914 Rio Xingú

Jan. 1915 Belém \& vicinity

June 1917 Rio Tapajós

TERr. AMApá

Nov. 1910 Ilha de Caviana

Dec. 1912 Rio Jari

Collections at MG

Ornithologist at Museu Goeldi, Belém

Made plant collections while on ornithological expeditions between 1905 \& 1919.

SPRUCE, RICHARD (1817 - 1893)

Amazonas; Pará

1849 - 1855

AMAZONAS

Oct. - 1850 - Nov. 1851 Ilha Tupinambarama, Manaus, lower Rio Solimões, lower Rio Negro

Nov. 1851 - Mar. 1853 Rio Negro, Barcelos, Santa Izabel, São Gabriel, Rio Uaupés, Panuré, Rio Negro, São Gabriel, Mari- 
bitanas; into Venezueia (Mar. 1853 about July 1854); return to Manaus

Dec. 1854 - Mar. 1855 Manaus

Mar. - May 1855 Manaus, Rio Solimões to Tabatinga and from there into Peru

PARÁ

June 1849 -Oct. 1850 Belém, Caripi, Tanaii, Rio Acará, Santarém, Óbidos, Rio Trombetas, Rio Aripecurú, Óbidos, Ilha Marimaritúba, Santarém, Rio Tapajós.

$\mathrm{K}, \mathrm{OXF}, \mathrm{BM}, \mathrm{BR}, \mathrm{CGE}, \mathrm{E}, \mathrm{F}, \mathrm{G}, \mathrm{GH}, \mathrm{GOET}, \mathrm{LD}$, LE,M,NY,OXF,P

Spruce collected about 7000 numbers some of which came from Venezuela Care should be taken in citing Spruce collections since many of his Venezuelan collections have often been cited as from Brazil since this is stated on the labels.

For further details see Urban (1906)

\section{STOUT}

1941 ?

Associated with the Chicle Development project; see Adams \& C.W. Smith. A very few collections bear Stout's name and were numbered in the Krukoff series, i.e. Nos 700-704; 12600-12601

SOUZA, R; see Royal Society

\section{TAKEUCHI, MASAYUKI}

\section{AMAZONAS}

$1958-1960$

1958 Amazonas; Manaus (3 collections)

Jan. 1960 Amazonas; Manaus (24 collections)

Feb. 1960 Amazonas; Lago do Janaur.cá (4 collections)

Deposited at INPA.

Collections without collector's number but with INPA herbarium number.

Takeuchi's visit to Amazonia was primarily for ecological research.

TAUBERT, PAUL HERMANN WILHELM

(1862 - 1897)

AMAZONAS

1896 Amazonas; Manaus \& vicinity
Died in Manaus January 1, 1897.

Collections from Brazil sold but present deposit unknown. Also collected in northeastern Brazil and Maranhão.

For obituary see Loesener, Th.; Verh. Bot. Ver. Brandenburg 39: LXII-LXVI. 1897.

\section{TRAILL, JAMES WILLIAM HELENUS}

(1851 - 1919)

AMAZONAS; PaRÁ

1873 - 1875

Amazonas 1874 Serra de Parintins, Faro, Maués, Rio Abacaxis, Rio Madeira, Borba, Manicore, Três Casas, Humaitá, return down river to Manaus, Rio Negro, Airão, Barcelos, Santa Izabel, Rio Padauiri, Mapapurá, return to Manaus, Rio Solimões, Manacapurú, Rio Purus, Paricatuba, Tauariá, Tapua, Lábrea, \& return to Rio Solimões, Coari, Tefé, Parauarí, Uará, mouth of Rio Juruá, Fonte Boa, São Paulo de Olivença, Tabatinga, Rio Javari, return to Manaus.

1875 Manaus, Rio Juta.i

PARÁ

1875 Almeirím, Belém

1873 Belém, Monte Alegre. Ereré, Rio Maicurú, Serra de Tajuré, Rio Jauarí, Santarém

1874 Santarém; Rio Tapajós, Alter do Chão, Óbidos, Rio Trombetas, Lago? Caipurú, Rio Cuminá, Óbidos, Rio Tapajós.

Specimens at $\mathrm{K}$, fairly small collection, also collected insects \& other animals.

For further details see Urban (1906).

\section{TRESSEL}

PARÁ

1963

Sept.-Oct. 1963; Pará; Santarém, Curuá-Una. Nos. 1-22

Deposited at INPA. 
TRINTA, Z.A.; see Fromm, E.

ULE, ERNST HEINRICH GEORG (1854-1915)

AMAZONAS; TERR, RORAIMA; ACRE

$1900-1912$

July 1900-1902 Amazonas; Manaus, Rio Juruá, Marari, São Rumão, Bom F'im. Santa Clara, Itapoana, Manaus, Rio Juruá, Manaus, Rio Madeira to Santa Maria dos Marmelos, Rio Marmelos, Rio Branco, Manaus, Rio Solimões to Tabatinga and on to Peru.

Dec. 1909 Amazonas; Rio Negro, Manaus

Dec. 1910-Feb. 1912 Amazonas; Rio Purus, Bôca do Acre

Jan, - Feb. 1910 Terr. Roraima; Rio Branco to Mount Roraima

Dec. 1910-Feb. 1912 Acre; Rio Acre, Rio Branco, São Francisco to Peru and re. turn to Manaus the same way.

The first set of Ule collections was lost at Berlin during World War II. The best set is at Hamburg which has Ule numbers up to 7575 only. Duplicates of Ule collections are at $\mathrm{K}, \mathrm{L}, \mathrm{MG}, \mathrm{R}$.

For Obituary see : Harms, H. Be". Deut. Bot. Gesell. 33 (Schluss - Heft) : 52-59. 1916.

\section{ULISEES}

TERR, RONDÔNIA

1962

May 1962 Terr. Rondônia; Pôrto Velho

9 collections without collector's number, but with INPA herbarium numbers; deposited at INPA \& collected with L. Coêlho.

VALLE, RUBEM

\section{AMAZONAS}

1955

July 1955 Amazonas; Manaus

7 collections of Fungi without collector's number, one set deposited at INPA.
VASCONCELLS, C.T.

ACRE

1962

Feb. 1962 Acre; Rio Branco, Colônia Penal Agrícola (36 collections)

Feb. 1962 Acre; Rio Branco, Colônia Apo'onia Sales (16 collections)

Féb. 1962 Acre; Rio Branco, Colônia Seringal Emprêsa (12 collections)

Feb. 1962 Acre; Rio Brancc. Colônia Dias Martins (13 collections)

Fcb. 1962 Acre; Rio Branco, Colônia Cruz Milagrosa ( 8 collections)

Feb. 1962 Acre; Rio Branco, Colônia Cecília Parente (10 collections)

Feb. 1962 Acre; Rio Branco, Colônia Custódio Freire (21 collections)

Feb. 1962 Acre; Rio Branco, Colônia Juarez Távora (12 collections)

Feb. 1962 Acre; Rio Branco, Airport (22 collections)

Feb. 1962 Acre; Rio Branco, (30 collections)

Collections without collector's number but with INPA herbarium numbers. All collections made with D. Coêlho and deposited at INPA.

VIERA, ANTONIO NETO

AMazonas

1967

1967 Amazonas; Manaus

2 collections with INPA numbers deposited at INPA.

VCGEL, STEPHAN

Amazonas

1964

Dac.1964 Amazonas; Uaupés Nos. 331-365

Specimens deposited at INPA.

WALLACE, ALFRED RUSSELL (1822 - 1913)

AMAZONAS; PARÁ

$1848-1852$

AMAZONAS

1849 Manaus

1850 Rio Solimões, Rio Negro

1851 - 1852 Rio Negro 
PARÁ

1848 Rio Tocantins

1849 Iiha do Marajó, Santarém, Óbłdos.

Wallace's entire herbarium collection was lost in a ship fire and only his writings remain. For this reason only a brief summary of his extensive itinerary is given. For further details see Wallace, A.R. A narrative of travels on the Amazon and Rio Negro London (1853), and also Urban (1906).

WALITS, GUSTAV (1830 - 1878)

AMAZONAS; TERR, RORAIMA, PARÁ

$1863-1865$

A.MAZONAS

1860 - 1862 Rio Madeira, Rio Purus

1863 - 1864 Rio Negro

1865 Rio Solimões to Tabatinga and into Peru

TERR, RORAIMA

1863 - 1864 Rio Branco, Rio 'Tacutu, Rio Pa. rima, Serra Pacaraima.

PARÁ

1860 Rio Tapajós

Wallis made very few herbarium collections since he mainly collected living material. His herbarium material was destroyed at Berlin in World War II.

WEDDELL, HUG ALGERNON (1819 - 1877)

MATO GROSSO

$1844-1845$

Nov. - Dec. 1844 Mato Grosso; Rio Araguaia to Cuiabá, Diamantino

1845 Mato Grosso; Cuiabá; Rio Cuiabá, Rio Paraguai, Rio Jaurú, Cuiabá, Pocono, Rio Jaurú, Rio Guaporé, Mato Grosso, Cesalvasco, Rio Barbudo into Bolivia. $\mathrm{P}, \mathrm{G}$

WEISS, LOUIS

\section{AMAZONAS}

$1907-1908$

$\mathrm{NY}$

Made a small collection in the Upper Rio Negro accompanied by Herman Schmidt. So far I have been unable to find full details of their itinerary and collections, but apparently they collected mainly Cryptogams.

357 Brazilian mosses and 10 Fungi from the Cairi River Region were accessed at NY.

WESTRA, LUBERT Y. Th.

TERR, AMAPÁ

1960

Aug. - Oct. 1960 Terr. Amapá; Rio Oiapoque (Nos. 47297-47322, 48508-48556)

NY,F,GH,K,U,US

The numbers are New York Botanical Garden series. He also collected with Irwin n Amapá in the same number series.

\section{WICKHAM, SIR HENRY ALEXANDER}

PARÁ

$1871-1876$

PARÁ; Sentarém, Rio Tapajós

$\mathrm{K}$

Collected some herbarium material while living at Santarém; but best known for the 70,00 seeds of rubber, Hevea braziliensis, which he collected and exported to Kew, England and ultimately Asia thus ending the South American monopoly in rubber, and causing cha.os to the economy of Amazônia.

WILLIAMS, LLEWELYN (1901 -

AMAZONAS; PARÁ; TERR. AMAPÁ

1955 - 1956

1956 Amazonas; Manaus \& vicinity

1956 Pará; Belém, Ilha do Marajó

Jan. Apr. 1955 Pará; Portel, Rio Pacajá, Rio Jacumda

1956 Terr. Amapá; Macapá.

IAN,US

Williams' visits to Brazilian Amazonia were for the study of Manilkara and other latex yielding trees, hence his collections are largely of those. Materials of the latex trees are still with Williams. Some duplicates $O_{\text {. }}$ material have been distributed. Williams also collected widely in Peru \& Venezuela. 
WILSON - Albuquerque, B.W. de

WURDACK, JOHN JULIUS (1921 -

PARÁ

1956

Dec. 1956 Pará; Serra do Cachimbo

Visited Serra do Cachimbo, Pará together with J.M. Pires \& G.A. Black. Plants from this expedition distributed under Pires numbers 6062-6556.
Also collected in Rio Grande do Norte and widely in Venezuela.

\section{ZERNY, H.}

PARÁ

1927

PArÁ; Belém, Santarém, Rio Tapajós

An entomologist who was primarily concerned with insect collections; however, he made a few collections from Pará which are

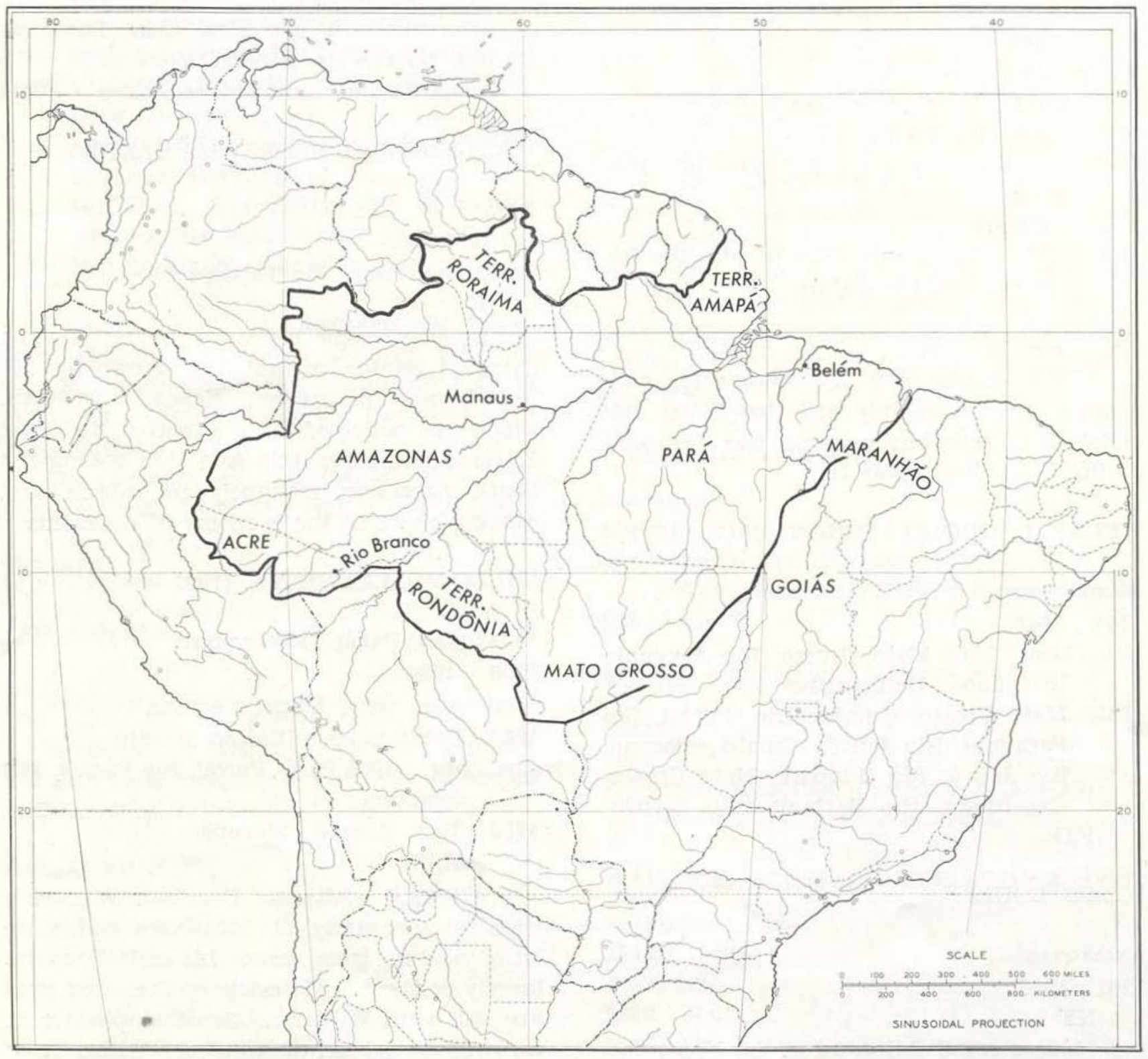

Fig. : - Map of area covered by Index. 
deposited at W. Also accompanied A. Ginzberger on his botanical work in the region

PRINCIPAL PUBLISHED GENERAL SOURCES OF COLLECTORS INFORMATION

ANGELY, J .

1959 - Index dos coletores para a flora do Brasil; 1a Parte: A-H. Bol. InFaBo $10: 1-15$.

BARNHART, H. J.

1965 - Biographical notes upon hotanists complied by John Hendley Barnhart and maintained in the New York Botanical Garden, Boston.
De Candolle, A. L. P. P.

- La Phytographie ou L'art de décrire les végétaux considérés sous différents points de vue... Paris . .

Lanjouw, J. \& F. A. Stafleu

1954, 1957 - Index Herbaiarum. Part 2, Nos, 1 \& 2. Corlecters A D \& E-H. Regnum Vegetabile $2 \& 9$.

URBAN, I.

1906 - Vitae Itineraque collectorum Botanicorum. In Martius, K. F. P. von, Flora Brasiliensis, $1(1): 1-152$.

\section{No próximo número:}

\section{BOTÂNICA -}

Micranda scleroxyla, nova Euforbiácea da Amazonia Brasileira - W.A. Rodrigues.

Notas sôbre os gêneros Polygonanthus e Anisophyllea - J.M. Pires e W.A. Rodrigues.

Nôvo Phyllanthus (Euforbiaceae) da Amazô nia Brasileira - W.A. Rodrigues.

Os TIPOS no herbário do Instituto Nacional de Pesquisas da. Amazônia - M.A. Freitas da Silva.

\section{FITOQUIMICA -}

The chemical composition of Amazon Plants - II - Setor de Fitoquímica do INPA.

\section{CIENCIAS DO AMBIENTE -}

Air temperatures in Central Amazonia II The effect of near surface temperatures on land "use in the Tertiary Region of Central Amazonia - W.L.F. Brinkmann e M.N. Góez Ribeiro .
Light environments on Tropical Rain Forest of Central Amazonia - W.L.F. Brinkmann.

\section{PATOLOGIA TROPICAL -}

Sobre a infecção natural do Panstrongylus geniculatus por Trypanosoma cruzi em Manaus - F.B. Almeida e P.A. Machado.

\section{TECNOLOGIA -}

Madeiras denominadas Ucuuba I - Virola divergens Ducke e V. multinerva Ducke M. Honda .

Contribuição ao estudo anatômico da madeira de Anonáceas da Amazônia III - Annona sericea Dun, A. poludosa Aubl e Gratteria paraensis RE Fries - A.A. Loureiro.

DEPOIMENTO DE VISITANTES - My experience in Amazonia - J. H. Langenheim. 